\title{
The Jones polynomial of ribbon links
}

\author{
MiCHAEL EISERMANN
}

For every $n$-component ribbon link $L$ we prove that the Jones polynomial $V(L)$ is divisible by the polynomial $V\left(\bigcirc^{n}\right)$ of the trivial link. This integrality property allows us to define a generalized determinant det $V(L):=\left[V(L) / V\left(\bigcirc^{n}\right)\right]_{(t \mapsto-1)}$, for which we derive congruences reminiscent of the Arf invariant: every ribbon link $L=K_{1} \cup \cdots \cup K_{n}$ satisfies $\operatorname{det} V(L) \equiv \operatorname{det}\left(K_{1}\right) \cdots \operatorname{det}\left(K_{n}\right)$ modulo 32, whence in particular det $V(L) \equiv 1$ modulo 8 .

These results motivate to study the power series expansion $V(L)=\sum_{k=0}^{\infty} d_{k}(L) h^{k}$ at $t=-1$, instead of $t=1$ as usual. We obtain a family of link invariants $d_{k}(L)$, starting with the link determinant $d_{0}(L)=\operatorname{det}(L)$ obtained from a Seifert surface $S$ spanning $L$. The invariants $d_{k}(L)$ are not of finite type with respect to crossing changes of $L$, but they turn out to be of finite type with respect to band crossing changes of $S$. This discovery is the starting point of a theory of surface invariants of finite type, which promises to reconcile quantum invariants with the theory of Seifert surfaces, or more generally ribbon surfaces.

57M25; 57M27

\section{Introduction}

It is often lamented that, after more than 20 years of intense research and spectacular success, we still do not have a good topological understanding of the Jones polynomial. This is in sharp contrast to the Alexander polynomial: to mention just one prominent example (Fox-Milnor [15]), the Alexander polynomial $\Delta(K)$ of every ribbon or slice knot $K$ has a beautiful and very strong symmetry, $\Delta(K)=f(q) \cdot f\left(q^{-1}\right)$, whereas no similar result is known for the Jones polynomial.

Only a few special values of the Jones polynomial have a topological interpretation, most notably the determinant $\operatorname{det}(L)=\left.V(L)\right|_{(q \mapsto i)}=\left.\Delta(L)\right|_{(q \mapsto i)}$. (See Section 2 for definitions; we use the parameterization $t=q^{2}$ throughout.) 


\subsection{Statement of results}

We define the nullity null $V(L)$ of the Jones polynomial $V(L)$ to be the multiplicity of the zero at $q=i$. The trivial link with $n$ components, for example, satisfies $V\left(\bigcirc^{n}\right)=\left(q^{+1}+q^{-1}\right)^{n-1}$ and thus null $V\left(\bigcirc^{n}\right)=n-1$.

Lemma 1 The Jones nullity equals the multiplicity of the factor $\left(q^{+1}+q^{-1}\right)$ in $V(L)$. If $L$ has $n$ components, then $\left.V(L)\right|_{(q \mapsto 1)}=2^{n-1}$ and thus $0 \leq$ null $V(L) \leq n-1$.

This inequality provides a first piece in the puzzle: the same bounds $0 \leq \operatorname{null}(L) \leq n-1$ hold for Murasugi's nullity derived from the Seifert form; see Section 2 for details.

Proposition 1 Consider a link $L \subset \mathbb{R}^{3}$ bounding a properly embedded smooth surface $S \subset \mathbb{R}_{+}^{4}$ without local minima. If $S$ has positive Euler characteristic $n=\chi(S)$, then the Jones polynomial $V(L)$ is divisible by $V\left(\bigcirc^{n}\right)=\left(q^{+}+q^{-}\right)^{n-1}$ and so null $V(L) \geq n-1$.

The condition is equivalent to saying that $L$ bounds an immersed surface $S \subset \mathbb{R}^{3}$ of Euler characteristic $n$ and having only ribbon singularities; see Section 3 for details.

Again the same inequality, null $(L) \geq \chi(S)-1$, holds for the Seifert nullity. Upper and lower bound for null $V(L)$ coincide precisely for ribbon links.

Theorem 1 Every $n$-component ribbon link $L$ satisfies null $V(L)=n-1$.

This corresponds to the Seifert nullity, so we see that null $(L)=$ null $V(L)$ for every ribbon link $L$. It would be interesting to know whether this equality generalizes to all links, see Question 7.8 and Question 7.9 towards the end of this article.

Expanding $V(L)$ in $q=\exp (h / 2)$ we obtain a power series $V(L)=\sum_{k=0}^{\infty} v_{k}(L) h^{k}$ whose coefficients $v_{k}(L)$ are link invariants of finite type in the sense of Vassiliev [46] and Goussarov [19], see also Birman-Lin [4] and Bar-Natan [2]. The above results motivate to study the power series expansion $V(L)=\sum_{k=0}^{\infty} d_{k}(L) h^{k}$ in $q=$ $i \exp (h / 2)$. We obtain a family of invariants $d_{k}(L)$ starting with the link determinant $d_{0}(L)=\operatorname{det}(L)$. The Jones nullity null $V(L)$ is the smallest index $v$ such that $d_{v}(L) \neq$ 0 . The link invariants $d_{k}(L)$ are not of finite type with respect to crossing changes. They enjoy, however, the following surprising property.

Proposition 2 The surface invariant $S \mapsto d_{k}(\partial S)$ is of finite type with respect to band crossing changes. More precisely, it is of degree $\leq m$ for $m=k+1-\chi(S)$. 
See Section 5.5 for definitions. The only invariant of degree $<0$ is the zero map: for $k<\chi(S)-1$ we thus have $d_{k}(\partial S)=0$ as in Proposition 1. The case $m=0$ corresponds to $k=\chi(S)-1$; being of degree $\leq 0$ means that $d_{k}(\partial S)$ is invariant under band crossing changes. Specializing to ribbon links we obtain the following result.

Corollary 2 For every $n$-component ribbon link $L=K_{1} \cup \cdots \cup K_{n}$ the Jones determinant det $V(L):=\left[V(L) / V\left(\bigcirc^{n}\right)\right]_{(q \mapsto i)}$ is invariant under band crossing changes. If $L$ bounds an immersed ribbon surface $S \subset \mathbb{R}^{3}$ consisting of $n$ disjoint disks, then we have det $V(L)=\operatorname{det}\left(K_{1}\right) \cdots \operatorname{det}\left(K_{n}\right)$, whence $\operatorname{det} V(L)$ is an odd square integer.

For a ribbon surface $S \subset \mathbb{R}^{3}$ consisting of disks which may intersect each other, multiplicativity only holds modulo 32 , and examples show that this is the best possible value.

Theorem 2 Every $n$-component ribbon link $L=K_{1} \cup \cdots \cup K_{n}$ satisfies $\operatorname{det} V(L) \equiv$ $\operatorname{det}\left(K_{1}\right) \cdots \operatorname{det}\left(K_{n}\right)$ modulo 32 , and in particular $\operatorname{det} V(L) \equiv 1$ modulo 8 .

These results can be seen as a first step towards understanding the Jones polynomial of ribbon links. It is plausible to expect that our results can be extended in several ways, and we formulate some natural questions in Section 7. As an application, Theorem 1 is used in Eisermann-Lamm [11] as an integrality property of the Jones polynomial of symmetric unions.

\subsection{Related work}

Little is known about the Jones polynomial of ribbon knots, but there is strong evidence that the expansion at $t=-1$ (that is, $q=i$ ) plays a crucial rôle.

First of all, for every ribbon knot $K$, the determinant $d_{0}(K)=\operatorname{det}(K)=V(K)_{t \mapsto-1}$ is a square integer, see Remark 3.6, and the resulting congruence $\operatorname{det}(K) \equiv 1 \bmod 8$ is related to the Arf invariant of knots, see Lickorish [32, chapter 10].

Next, the first-order term $d_{1}(K)=-\left[\frac{d}{d t} V(K)\right]_{t \mapsto-1}$ figures prominently in the work of Mullins [37, Theorem 5.1], who discovered a beautiful relation with the Casson-Walker invariant $\lambda\left(\Sigma_{K}^{2}\right)$ of the 2 -fold branched cover of $\mathbb{S}^{3}$ branched along $K$ :

$$
\lambda\left(\Sigma_{K}^{2}\right)=\frac{1}{4} \operatorname{sign}(K)-\frac{1}{6}\left[\frac{\frac{d}{d t} V(K)}{V(K)}\right]_{t \mapsto-1}=\frac{1}{4} \operatorname{sign}(K)+\frac{1}{6} \frac{d_{1}(K)}{d_{0}(K)} .
$$


This identity holds for every knot $K \subset \mathbb{S}^{3}$, and more generally for every link with non-vanishing determinant. Garoufalidis [17, Theorem 1.1] generalized Mullins' result to all links, using the Casson-Walker-Lescop invariant $\Lambda$ constructed by Lescop [30]:

$$
i^{[\operatorname{sign}(K)+\operatorname{null}(K)]} \cdot \Lambda\left(\Sigma_{K}^{2}\right)=\frac{1}{4} d_{0}(K) \operatorname{sign}(K)+\frac{1}{6} d_{1}(K) .
$$

If $\operatorname{det}(K) \neq 0$, then $\Sigma_{K}^{2}$ is a rational homology sphere; in this case the Casson-Walker invariant is defined and satisfies $\lambda\left(\Sigma_{K}^{2}\right) \cdot|\operatorname{det}(K)|=\Lambda\left(\Sigma_{K}^{2}\right)$, so that (2) implies (1).

If $\operatorname{det}(K)=1$, then $\Sigma_{K}^{2}$ is an integral homology sphere and $\lambda\left(\Sigma_{K}^{2}\right) \in \mathbb{Z}$ is Casson's original invariant. If, moreover, $K$ is a ribbon knot, then $\operatorname{sign}(K)$ vanishes and $\lambda\left(\Sigma_{K}^{2}\right)$ is an even integer because it reduces modulo 2 to the Rohlin invariant and $\Sigma_{K}^{2}$ bounds a homology 4-ball, see Casson-Gordon [6, Lemma 2]. In this case $d_{1}(K)$ is divisible by 12 . No such congruences seem to be known for higher order terms $d_{2}, d_{3}, \ldots$, nor for ribbon knots or links in general.

Generalizing work of Sakai, Mizuma has worked out an explicit formula for $d_{1}(K)$ of 1 -fusion ribbon knots $K$ [35] and derived a lower bound for the ribbon number [36].

Studying link concordance, Cochran [7, Corollary 3.10] has established similar properties and congruences for the first non-vanishing coefficients of the Conway polynomial.

\subsection{How this article is organized}

Theorem 1 and Theorem 2 are pleasant to state but their proofs are somewhat technical: we proceed by induction on planar diagrams of immersed surfaces in $\mathbb{R}^{3}$. The arguments are elementary but get increasingly entangled. Generally speaking, these technicalities are due to the combinatorial definition of the Jones polynomial whereas the ribbon condition is topological in nature.

The article follows the outline given in this introduction. Section 2 recollects some basic definitions and highlights motivating analogies; the upper bound of Lemma 1 is derived from Jones' skein relation by an algebraic argument. In order to apply skein relations to ribbon links, Section 3 recalls the notions of slice and ribbon links, and introduces planar band diagrams as a convenient presentation. Section 4 sets up a suitable induction technique for the Kauffman bracket and proves the lower bound of Proposition 1. Section 5 discusses band crossing changes and proves Proposition 2. Section 6 establishes multiplicativity modulo 32 as stated in Theorem 2. Section 7, finally, discusses possible generalizations and open questions. 


\subsection{Acknowledgements}

I thank Christoph Lamm for countless inspiring discussions, which sparked off the subject. I am also indebted to Christine Lescop, Thomas Fiedler and Christian Blanchet for valuable suggestions and comments on successive versions of this article. This work was begun in the winter term 2006/2007 during a sabbatical funded by a research contract délégation auprès $d u C N R S$, whose support is gratefully acknowledged.

\section{Definitions and first properties}

The nullity and the determinant that we introduce and study for the Jones polynomial are analogous to the corresponding notions of the classical Seifert form. In order to highlight these intriguing analogies most convincingly, we shall review side by side some elementary properties of the Alexander-Conway and the Jones polynomial.

As standard references in knot theory we refer to Burde-Zieschang [5], Lickorish [32] and Rolfsen [44]. Throughout this article we work in the smooth category.

\subsection{The Alexander-Conway polynomial}

We denote by $\mathbb{Z}\left[q^{ \pm}\right]$the ring of Laurent polynomials in the variable $q=q^{+}$with inverse $q^{-1}=q^{-}$. Its elements will simply be called polynomials in $q$. For every link $L \subset \mathbb{R}^{3}$ we can construct a Seifert surface $S$ spanning $L$, that is, a compact connected oriented surface $S \subset \mathbb{R}^{3}$ such that $L=\partial S$ with induced orientations. We choose a basis of $H_{1}(S)$ and denote by $\theta$ and $\theta^{*}$ the associated Seifert matrix and its transpose, respectively; see Burde-Zieschang [5, Definition 8.5], Lickorish [32, Definition 6.5] or Rolfsen [44, Definition 8A1]. The Alexander-Conway polynomial $\Delta(L) \in \mathbb{Z}\left[q^{ \pm}\right]$is defined as $\Delta(L)=\operatorname{det}\left(q^{-} \theta^{*}-q^{+} \theta\right)$. It does not depend on the choice of $S$ and is thus an isotopy invariant of the link $L$. It is traditionally parameterized by $t=q^{2}$, but we prefer the variable $q=-t^{1 / 2}$ in order to avoid square roots and to fix signs.

We denote by $\mathbb{R}$ the set of isotopy classes of oriented links $L \subset \mathbb{R}^{3}$. The map $\Delta: \mathfrak{R} \rightarrow \mathbb{Z}\left[q^{ \pm}\right]$is characterized by Conway's skein relation

$$
\Delta(\swarrow)-\Delta(\searrow)=\left(q^{+}-q^{-}\right) \Delta()()
$$

with the initial value $\Delta(\bigcirc)=1$. The skein relation entails that $\Delta(L \sqcup \bigcirc)=0$.

The (signed) determinant of a link is defined as

$$
\operatorname{det}(L):=\left.\Delta(L)\right|_{(q \mapsto i)}=\operatorname{det}\left[-i\left(\theta+\theta^{*}\right)\right] .
$$


Most authors consider the determinant $\operatorname{det}\left(\theta+\theta^{*}\right)$, but then only its absolute value $\left|\operatorname{det}\left(\theta+\theta^{*}\right)\right|$ is invariant; see Burde-Zieschang [5, Corollary 13.29], Lickorish [32, page 90], Rolfsen [44, Definition 8D4]. In our normalization the determinant is the unique invariant det: $\mathbb{R} \rightarrow \mathbb{Z}[i]$ that satisfies $\operatorname{det}(\bigcirc)=1$ and the skein relation

$$
\operatorname{det}(\swarrow)-\operatorname{det}(\searrow)=2 i \operatorname{det}()() \text {. }
$$

\subsection{Signature and nullity}

Murasugi [40] showed that the signature $\operatorname{sign}(L):=\operatorname{sign}\left(\theta+\theta^{*}\right)$ and the nullity $\operatorname{null}(L):=\operatorname{null}\left(\theta+\theta^{*}\right)$ are invariants of the link $L$. Tristram [45] generalized this by passing from the symmetric matrix $\theta+\theta^{*}$ to the hermitian matrix

$$
M_{\omega}:=\left(1-\omega^{2}\right) \theta+\left(1-\bar{\omega}^{2}\right) \theta^{*}=(\omega-\bar{\omega})\left(\bar{\omega} \theta^{*}-\omega \theta\right)
$$

with $\omega \in \mathbb{S}^{1} \backslash\{ \pm 1\}$. He showed that the generalized signature $\operatorname{sign}_{\omega} L:=\operatorname{sign} M_{\omega}$ and the generalized nullity null $\omega:=$ null $M_{\omega}$ are again link invariants. Independently, Levine [31] defined the same invariants for knots. For $\omega= \pm i$ this specializes to Murasugi's invariants. For higher dimensions see Erle [12] and Milnor [34].

Remark 2.1 For every knot $K$ we have $\operatorname{det}(K) \equiv 1 \bmod 4$, whence $\operatorname{det}(K) \neq 0$ and $\operatorname{null}(K)=0$. More generally, let $N \in \mathbb{N}$ be a prime number and let $\omega$ be a primitive $2 N$ th root of unity. Tristram [45, Lemma 2.5] remarked that the generalized determinant

$$
\operatorname{det}_{\omega}(L):=\left.\Delta(L)\right|_{(q \mapsto \omega)}=\operatorname{det}\left(\bar{\omega} \theta^{*}-\omega \theta\right)
$$

never vanishes for a knot. More generally, he proved that $0 \leq \operatorname{null}_{\omega}(L) \leq n-1$ for every link $L$ with $n$ components [45, Corollary 2.24]. We shall see below that the same technique applies to the Jones polynomial.

For the matrix $M:=q^{-} \theta^{*}-q^{+} \theta$ over $\mathbb{Z}\left[q^{ \pm}\right] \subset \mathbb{Q}(q)$, the nullity null $M$ is the dimension of its null-space. We have null $M \leq$ null $M_{\omega}$ for all $\omega \in \mathbb{S}^{1}$, and equality holds for all but finitely many values of $\omega$. In particular we see that $0 \leq$ null $M \leq n-1$.

\subsection{The Jones polynomial}

The following theorem is due to Alexander [1] and Conway [8] for $N=0$, Jones [21] for $N=2$, and HOMFLYPT (Freyd-Yetter-Hoste-Lickorish-Millett-Ocneanu [16], Przytycki-Traczyk [42]) for the general case $N \in \mathbb{N}$.

Theorem 2.2 For each $N \in \mathbb{N}$ there exists a unique link invariant $V_{N}: \mathfrak{R} \rightarrow \mathbb{Z}\left[q^{ \pm}\right]$ mapping the trivial knot to $V_{N}(\bigcirc)=1$ and satisfying the following skein relation:

$$
q^{-N} V_{N}(\swarrow)-q^{+N} V_{N}(\searrow)=\left(q^{-1}-q^{+1}\right) V_{N}()() \text {. }
$$


The case $N=0$ reproduces the Alexander-Conway polynomial, $V_{0}(L)=(-1)^{n-1} \Delta(L)$ where $n$ is the number of components. The choice $N=1$ yields the trivial invariant, $V_{1}(L)=1$ for all $L \in \mathbb{R}$. The case $N=2$ yields the Jones polynomial [21], traditionally parameterized by $t=q^{2}$ with the sign convention $q=-t^{1 / 2}$ (see Section 4.1).

Remark 2.3 It follows from these axioms that $V_{N}(L \sqcup \bigcirc)=V_{N}(L) \cdot U_{N}$ with

$$
U_{N}=\frac{q^{-N}-q^{+N}}{q^{-1}-q^{+1}} .
$$

We have $U_{0}=0$ and $U_{1}=1$, while for $N \geq 2$ we obtain the expansion

$$
U_{N}=q^{-N+1}+q^{-N+3}+\cdots+q^{N-3}+q^{N-1} .
$$

This is sometimes called the quantum integer $[N]_{q}$. For $q \mapsto 1$ we get $[N]_{(q \mapsto 1)}=N$. For the trivial $n$-component link we have $V_{N}\left(\bigcirc^{n}\right)=U_{N}^{n-1}$, and for every $n$-component link $L$ we obtain

$$
\left.V_{N}(L)\right|_{(q \mapsto 1)}=\left.V_{N}\left(\bigcirc^{n}\right)\right|_{(q \mapsto 1)}=N^{n-1} .
$$

Finally, we observe the following symmetry with respect to the automorphism $q \mapsto-q$, which corresponds to the non-trivial Galois automorphism of $\mathbb{Z}\left[t^{ \pm 1 / 2}\right]$ over $\mathbb{Z}\left[t^{ \pm}\right]$:

$$
\left.V_{N}(L)\right|_{(q \mapsto-q)}=(-1)^{(N-1)(n-1)} V_{N}(L) .
$$

If $N$ is odd, then $V_{N}(L)$ is even, that is, invariant under the automorphism $q \mapsto-q$. If $N$ is even, then $\left.V_{N}(L)\right|_{(q \mapsto-q)}=(-1)^{n-1} V_{N}(L)$ depends on the parity of $n$.

\subsection{An upper bound for the Jones nullity}

We are now ready to prove Lemma 1 . The idea is to adapt Tristram's observation [45, Lemma 2.5] to the Jones polynomial.

Definition 2.4 The nullity null $z=v$ of a Laurent polynomial $P \in \mathbb{C}\left[q^{ \pm}\right]$at some point $z \in \mathbb{C} \backslash\{0\}$ is the multiplicity $v$ of the root at $q=z$.

More explicitly, we have $P=(q-z)^{v} \cdot Q$ such that $v \geq 0$ and $Q \in \mathbb{C}\left[q^{ \pm}\right]$satisfies $Q(z) \neq 0$. Alternatively, $v$ is the least integer such that the derivative $P^{(v)}=\frac{d^{v}}{d q^{v}} P$ does not vanish in $z$. It is also the smallest index such that $d_{v} \neq 0$ in the power series expansion $P(q)=\sum_{k=0}^{\infty} d_{k} h^{k}$ at $q=z \exp (h / 2)$.

The polynomial $U_{N} \in \mathbb{Z}\left[q^{ \pm}\right]$of degree $2 N-2$ vanishes at every $2 N$ th root of unity $\omega$ other than \pm 1 , so that $\operatorname{null}_{\omega} U_{N}=1$. We fix a primitive $2 N$ th root of unity, $\omega=\exp (i \pi k / N)$, by specifying an integer $k$ such that $0<k<2 N$ and $\operatorname{gcd}(k, 2 N)=1$. 
Proposition 2.5 Let $L \subset \mathbb{R}^{3}$ be a link. If $N$ is prime, then we have the factorization $V_{N}(L)=U_{N}^{v} \cdot \tilde{V}_{N}(L)$ with $v=\operatorname{null}_{\omega} V_{N}(L)$ and $\tilde{V}_{N}(L) \in \mathbb{Z}\left[q^{ \pm}\right]$such that $U_{N} \nmid$ $\tilde{V}_{N}(L)$.

Proof If $N=2$, then $\omega= \pm i$, whence $q^{2}+1=q U_{2}$ is the minimal polynomial of $\omega$ in $\mathbb{Q}[q]$. For each $P \in \mathbb{Z}\left[q^{ \pm}\right]$, the condition $P(\omega)=0$ is equivalent to $P=U_{2} \cdot Q$ for some $Q \in \mathbb{Z}\left[q^{ \pm}\right]$. Iterating this argument, we obtain $P=U_{2}^{v} \cdot Q$ with $Q \in \mathbb{Z}\left[q^{ \pm}\right]$ such that $Q(\omega) \neq 0$, whence null $_{\omega} P=v$.

If $N$ is odd, then $\omega$ is of order $2 N$ and $-\omega$ is of order $N$ in the multiplicative group $\mathbb{C}^{\times}$. Their minimal polynomials in $\mathbb{Q}[q]$ are the cyclotomic polynomials $[29$, Section VI.3]

$$
\Phi_{2 N}=\prod_{\substack{\xi \in \mathbb{C}^{\times} \\ \operatorname{ord}(\xi)=2 N}}(q-\xi) \text { and } \Phi_{N}=\prod_{\substack{\xi \in \mathbb{C}^{\times} \\ \operatorname{ord}(\xi)=N}}(q-\xi) .
$$

If moreover $N$ is prime, then all $2 N$ th roots of unity are either of order $1,2, N$, or $2 N$ and thus $q^{2 N}-1=(q-1)(q+1) \Phi_{N} \Phi_{2 N}$. This implies that

$$
\Phi_{N} \cdot \Phi_{2 N}=q^{2 N-2}+q^{2 N-4}+\cdots+q^{2}+1=q^{N-1} U_{N} .
$$

This polynomial is even, has integer coefficients and leading coefficient 1 . As a consequence, if $P \in \mathbb{Z}\left[q^{ \pm}\right]$is even, then $P(\omega)=0$ is equivalent to $P=U_{N} \cdot Q$ for some $Q \in \mathbb{Z}\left[q^{ \pm}\right]$, and $Q$ is again even. Iterating this argument, we obtain $P=U_{N}^{v} \cdot Q$ with $Q \in \mathbb{Z}\left[q^{ \pm}\right]$even and $Q(\omega) \neq 0$, whence null $\omega=v$.

Corollary 2.6 Let $N$ be a prime and let $\omega \neq \pm 1$ be a $2 N$ th root of unity. Then the nullity null $V_{N}(L)$ only depends on $N$ and will thus be denoted by null $V_{N}(L)$. For every link $L$ with $n$ components we have the inequality $0 \leq$ null $V_{N}(L) \leq n-1$.

Proof We have $V_{N}(L)=U_{N}^{v} \cdot \tilde{V}_{N}(L)$ with $v=\operatorname{null}_{\omega} V_{N}(L)$ and $\tilde{V}_{N}(L) \in \mathbb{Z}\left[q^{ \pm}\right]$. Evaluating at $q=1$, we find $N^{n-1}=\left.N^{v} \cdot \widetilde{V}_{N}(L)\right|_{(q \mapsto 1)}$, whence $v \leq n-1$.

Definition 2.7 In the notation of the previous proposition, we call $\tilde{V}_{N}(L)$ the reduced Jones polynomial and $\operatorname{det}_{\omega} V_{N}(L):=\left.\widetilde{V}_{N}(L)\right|_{(q \mapsto \omega)}$ the Jones determinant of $L$ at $\omega$. It depends on the chosen root of unity $\omega$ up to a Galois automorphism of the ring $\mathbb{Z}[\omega]$.

Remark 2.8 The family of invariants $V_{N}$ with $N \in \mathbb{N}$ can be encoded by the HoMFLYPT polynomial $P: \mathbb{Z} \rightarrow \mathbb{Z}(q, \ell)$ defined by $P(\bigcirc)=1$ and the skein relation

$$
\left.\ell^{-} P(\swarrow)-\ell^{+} P(\searrow)=\left(q^{-}-q^{+}\right) P() \zeta\right) \text {. }
$$


This implies that $P(L \sqcup \bigcirc)=P(L) \cdot U$ with $U=\frac{\ell^{-}-\ell^{+}}{q^{-}-q^{+}}$. Moreover, $P$ takes values in the subring $R:=\mathbb{Z}\left[q^{ \pm}, \ell^{ \pm}, U\right]$ and is invariant under the ring automorphism $(\ell \mapsto-\ell, q \mapsto-q)$.

By construction, the following diagram is commutative:

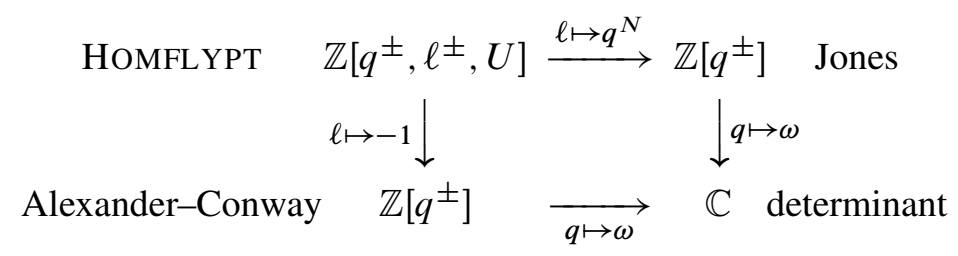

For every link $L$ we have a unique factorization $P(L)=U^{v} \cdot Q$ with $v \geq 0$ and $Q \in R$ satisfying $\left.Q\right|_{\ell= \pm 1} \neq 0$. We call null $P(L):=v$ the nullity of the HoMFLYPT polynomial. It satisfies the inequality null $P(L) \leq$ null $V_{N}(L)$ for all $N \in \mathbb{N}$, and equality holds for all but finitely many values of $N$. In particular $0 \leq$ null $P(L) \leq n-1$.

\section{Band diagrams for ribbon links}

\subsection{Band diagrams}

We wish to apply skein relations to ribbon links. To this end we shall use planar band diagrams built up from the pieces shown in Figure 1.

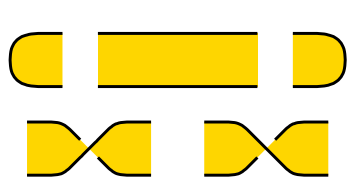

(a) ends, strip, twists

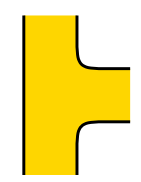

(b) band junction

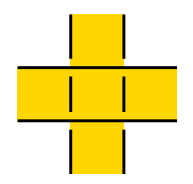

(c) band crossing

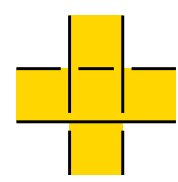

(d) ribbon singularity

Figure 1: Elementary pieces of band diagrams

Such a diagram encodes not only a link $L \subset \mathbb{R}^{3}$ but also an immersed surface $S \subset \mathbb{R}^{3}$ with boundary $\partial S=L$. More explicitly we have the following definition.

Definition 3.1 Let $\Sigma$ be a smooth compact surface with boundary $\partial \Sigma \neq \varnothing$. We do not require $S$ to be orientable nor connected, but we will assume that $S$ does not have any closed components. A smooth immersion $f: \Sigma \rightarrow \mathbb{R}^{3}$ is called (immersed) ribbon surface if its only singularities are ribbon singularities according to the local model shown in Figure 1d. Figure 2a displays a more three-dimensional view: every 


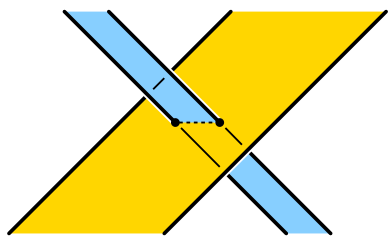

(a) a ribbon singularity

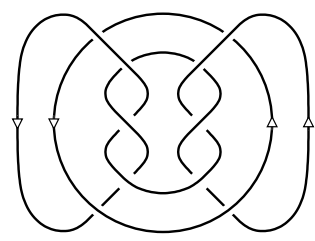

(b) a ribbon link $L$

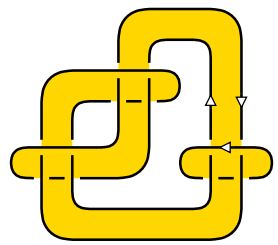

(c) ribbon disks for $L$

Figure 2: Ribbon links and immersed ribbon surfaces

component of self-intersection is an $\operatorname{arc} A$ so that its preimage $f^{-1}(A)$ consists of two $\operatorname{arcs}$ in $\Sigma$, one of which is interior.

Notation We regard $f$ only up to diffeomorphisms of $\Sigma$, and can thus identify the immersion $f$ with its image $S=f(\Sigma)$. The Euler characteristic $\chi(S)$ of the immersed surface $S$ is by definition the Euler characteristic of the abstract surface $\Sigma$. A component of $S$ is the image of a component of $\Sigma$.

A ribbon singularity is called mixed if it involves two distinct surface components. Otherwise, if the surface component pierces itself, the ribbon singularity is called pure.

We write $S=S_{1} \sqcup \cdots \sqcup S_{n}$ if the components $S_{1}, \ldots, S_{n}$ are contained in disjoint balls in $\mathbb{R}^{3}$. We also use the analogous notation $L=L_{1} \sqcup \cdots \sqcup L_{n}$ for links.

Since each surface component $S_{k}$ has non-empty boundary, it satisfies $\chi\left(S_{k}\right) \leq 1$. As a consequence, if a link $L$ has $n$ components, then every ribbon surface $S$ spanning $L$ satisfies $\chi(S) \leq n$. The maximum is attained precisely for ribbon links.

Definition 3.2 An $n$-component link $L \subset \mathbb{R}^{3}$ is called a ribbon link if it bounds a ribbon surface $S \subset \mathbb{R}^{3}$ consisting of $n$ disks. (Figure 2 shows an example.)

Proposition 3.3 For every band diagram $D$ there exists a ribbon surface $S \subset \mathbb{R}^{3}$ such that the standard projection $\mathbb{R}^{3} \rightarrow \mathbb{R}^{2}$ maps $S$ to $D$ in the obvious way.

Any two ribbon surfaces projecting to $D$ are ambient isotopic in $\mathbb{R}^{3}$. Modulo ambient isotopy we can thus speak of the surface realizing $D$,

Every ribbon surface $S \subset \mathbb{R}^{3}$ can be represented by a band diagram $D$, that is, $S$ is ambient isotopic to a surface $S^{\prime}$ projecting to $D$. 


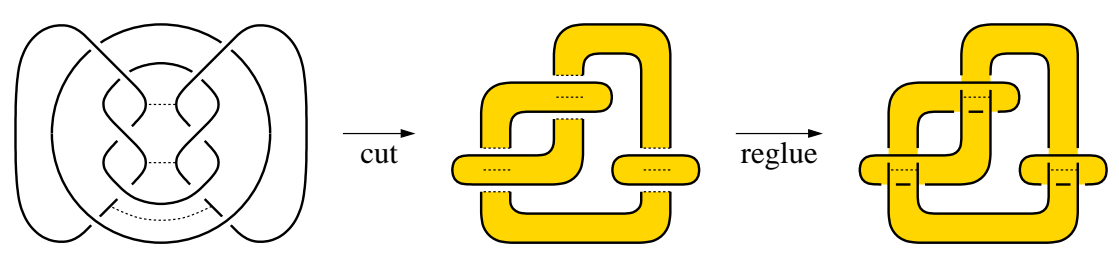

Figure 3: Cutting and regluing a ribbon surface

Proof We only sketch the last assertion: existence of a band diagram for every ribbon surface $S$. The idea is to cut $S$ along properly embedded arcs running from boundary to boundary. This is possible under our hypothesis that $S$ has no closed components. We repeat this process so as to obtain trivial pieces homeomorphic to disks.

These disks can be put disjointly into the plane and then reglued as prescribed; Figure 3 illustrates an example. Regluing typically introduces junctions, band crossings, twists and ribbon singularities; these suffice to achieve the reconstruction.

Remark 3.4 There is an analogue of Reidemeister's theorem, representing ambient isotopy of ribbon surfaces $S \subset \mathbb{R}^{3}$ by a generating set of local moves on band diagrams $D \subset \mathbb{R}^{2}$. The local moves are straightforward but lengthy to enumerate, and we shall not need this more precise result here. The general philosophy is that of links with extra structure, in our case links with a ribbon surface. This is an interesting topic in its own right, but we shall use it here merely as an auxiliary tool for our induction proof.

\subsection{Slice and ribbon links}

In order to put our subject matter into perspective, we briefly recall the 4-dimensional setting of slice and ribbon links.

We consider $\mathbb{R}^{3}$ as a subset of $\mathbb{R}^{4}$ via the standard inclusion $\left(x_{1}, x_{2}, x_{3}\right) \mapsto\left(x_{1}, x_{2}, x_{3}, 0\right)$. We say that a link $L \subset \mathbb{R}^{3}$ bounds a surface $S \subset \mathbb{R}_{+}^{4}=\left\{x \in \mathbb{R}^{4} \mid x_{4} \geq 0\right\}$ if $S$ is a properly embedded smooth surface such that $\partial S=S \cap \mathbb{R}^{3}=L$. (We will always assume that $S$ has no closed components.) A link $L$ is called slice if it bounds $n$ disjointly embedded disks in $\mathbb{R}_{+}^{4}$. This is sometimes called slice in the strong sense; see Fox [14] for a discussion of weaker notions. Slice knots naturally appear in the study of surfaces $\Sigma \subset \mathbb{S}^{4}$ with singularities, that is, isolated points where the surface $\Sigma$ is not locally flat, see Fox-Milnor [15], and Livingston [33] for a survey.

If an $n$-component link bounds a surface $S \subset \mathbb{R}_{+}^{4}$, then the Euler characteristic is bounded by $\chi(S) \leq n$, and the maximum is attained precisely for slice links. We are particularly interested in the case where the surface $S \subset \mathbb{R}_{+}^{4}$ has no local minima, 
or more explicitly, the height function $h: \mathbb{R}_{+}^{4} \rightarrow \mathbb{R}_{+}, x \mapsto x_{4}$, restricts to a Morse function $\left.h\right|_{S}$ without local minima. The following observation goes back to Fox [14].

Proposition 3.5 For every link $L \subset \mathbb{R}^{3}$ the following assertions are equivalent:

(1) $L$ bounds an immersed ribbon surface $S \subset \mathbb{R}^{3}$ such that $\chi(S)=n$.

(2) $L$ bounds a surface $S_{+} \subset \mathbb{R}_{+}^{4}$ without local minima such that $\chi\left(S_{+}\right)=n$.

As a consequence, $L$ is a ribbon link if and only if it bounds $n$ disjointly embedded disks in $\mathbb{R}_{+}^{4}$ without local minima. Whether all slice knots are ribbon is an open question which first appeared as Problem 25 in Fox's problem list [14]. Also see Problem 1.33 of Kirby's problem list [28].

Remark 3.6 Every $n$-component slice link $L$ satisfies $\operatorname{null}(L)=n-1$ and $\operatorname{sign}(L)=$ 0 (Murasugi [40]), and more generally $\operatorname{null}_{\omega}(L)=n-1$ and $\operatorname{sign}_{\omega}(L)=0$ where $\omega$ is a $2 N$ th root of unity and $N$ is prime (Tristram [45]). For $n=1$ the Alexander polynomial factors as $\Delta(L)=f\left(q^{+}\right) \cdot f\left(q^{-}\right)$with some $f \in \mathbb{Z}\left[q^{ \pm}\right]$(Fox-Milnor [15]). As a consequence $\operatorname{det}(K)$ is a square integer for every slice knot $K$, in particular $\operatorname{det}(K) \equiv 1 \bmod 8$. For $n \geq 2$, however, we have $\Delta(L)=0$, see Kawauchi [25]. It is a classical topic to study higher-order Alexander polynomials to remedy this problem; for the multi-variable Alexander polynomial see Kawauchi [25] and Florens [13]. We will instead look for extensions and analogies in the realm of quantum invariants.

\section{The Jones nullity of ribbon links}

Jones' skein relation (3) serves well for the upper nullity bound, but it turns out to be ill suited for the inductive proof that we shall be giving for the lower bound. We will thus prepare the scene by recalling Kauffman's bracket (Section 4.1). Ribbon link diagrams suggest a proof by induction, but one has to suitably generalize the statement (Section 4.2). I present here what I believe is the simplest induction proof, based on the Euler characteristic (Section 4.3).

\subsection{The Kauffman bracket}

The Kauffman bracket [23] is a map $\mathfrak{D} \rightarrow \mathbb{Z}\left[A^{ \pm}\right]$, denoted by $D \mapsto\langle D\rangle$, from the set D of unoriented planar link diagrams to the ring $\mathbb{Z}\left[A^{ \pm}\right]$of Laurent polynomials in the variable $A$. It is defined by the skein relation

$$
\begin{aligned}
\langle\backslash\rangle & \left.=A\langle\rangle(\rangle+A^{-1} \leftrightharpoons\right), \\
\langle D \sqcup \bigcirc\rangle & =\langle D\rangle \cdot\left(-A^{+2}-A^{-2}\right), \\
\langle\bigcirc\rangle & =1 .
\end{aligned}
$$


The bracket polynomial $\langle D\rangle$ is invariant under Reidemeister moves $\mathrm{R} 2$ and $\mathrm{R} 3$, called regular isotopy. Normalizing with respect to the writhe one obtains an isotopy invariant: upon the change of variables $q=-A^{-2}$ we thus recover the Jones polynomial

$$
\left.V(L)\right|_{\left(q=-A^{-2}\right)}=\langle D\rangle \cdot\left(-A^{-3}\right)^{\text {writhe }(D)} .
$$

Here $V(L)=V_{2}(L)$ is the Jones polynomial of the oriented link $L$, while $D$ is a planar diagram representing $L$, and $\langle D\rangle$ is its bracket polynomial (forgetting the orientation of $D$ ). The writhe of $D$ is the sum of all crossing signs.

Notation All subsequent calculations take place in the ring $\mathbb{Z}\left[A^{ \pm}\right] \supset \mathbb{Z}\left[q^{ \pm}\right] \supset \mathbb{Z}\left[t^{ \pm}\right]$ with $q=-A^{-2}$ and $t=q^{2}=A^{-4}$. This context explains the sign in $q=-t^{1 / 2}$ with $t^{1 / 2}=A^{-2}$ : although the roots $\pm t^{1 / 2}$ are conjugated in $\mathbb{Z}\left[t^{ \pm 1 / 2}\right]$ over $\mathbb{Z}\left[t^{ \pm}\right]$, this no longer holds in $\mathbb{Z}\left[A^{ \pm}\right]$. Choosing this convention I have tried to reconcile simplicity and tradition, so that all formulae become as simple as possible yet remain easily comparable. The results stated in the introduction are invariant under all possible normalizations and parameterizations, but of course such conventions are important in actual calculations and concrete examples.

\subsection{Proof strategy}

Applying Kauffman's skein relation to a ribbon singularity, we obtain the following 16 terms:

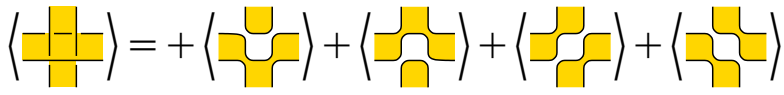

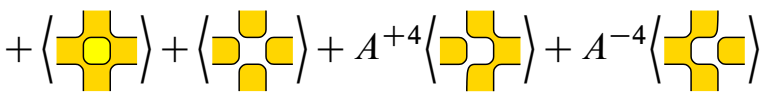

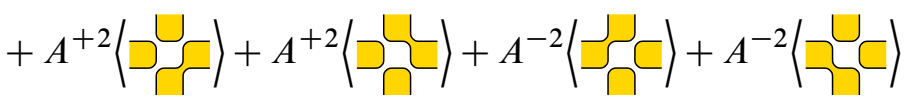

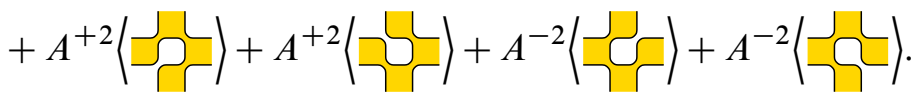


For a band crossing we obtain the same 16 terms with permuted coefficients:

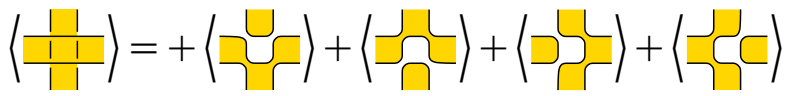

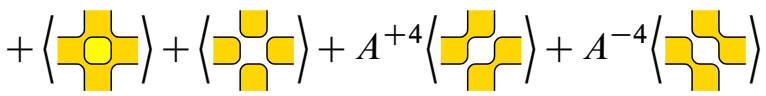

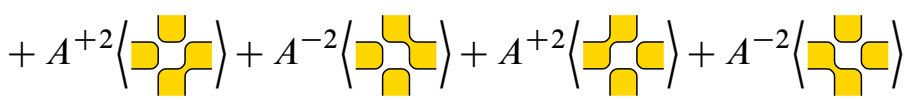

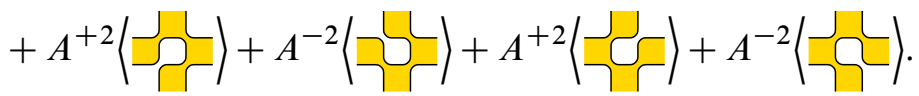

We are mainly interested in ribbon links, so we start out with a ribbon surface consisting only of disk components. Some of the resolutions displayed above, however, will lead to more complicated components, namely annuli or Möbius bands. We can avoid either Möbius bands or annuli by adding half twists as desired, but we cannot avoid both of them altogether. In order to set up an induction proof, this difficulty forces us to consider a suitable generalization including (at least) annuli or Möbius bands.

\subsection{A lower bound for the Jones nullity}

Even though we are primarily interested in ribbon links, we are obliged to prove a more general statement, as motivated above. The following seems to be the simplest setting supporting the desired inductive proof.

Proposition 4.1 If a link $L \subset \mathbb{R}^{3}$ bounds an immersed ribbon surface $S \subset \mathbb{R}^{3}$ of positive Euler characteristic $n$, then $V(L)$ is divisible by $V\left(\bigcirc^{n}\right)=\left(q^{+}+q^{-}\right)^{n-1}$.

Divisibility means that $V(L)=\left(q^{+}+q^{-}\right)^{n-1} \tilde{V}$ for some $\tilde{V} \in \mathbb{Z}\left[q^{ \pm}\right]$. In this formulation the proposition holds for all $n \in \mathbb{Z}$ but it is trivial, of course, for $n \leq 1$.

Example 4.2 The surface $S$ of Figure 4a has Euler characteristic $\chi(S)=1+1+0=2$, so for the link $L=\partial S$ we expect null $V(L) \geq 1$. Indeed we find null $V(L)=1$, because

$$
V(L)=\left(q^{+}+q^{-}\right) \cdot\left(q^{6}-q^{4}+2 q^{2}+2 q^{-2}-q^{-4}+q^{-6}\right) .
$$

We also remark that $L=8 n 8$ is the (anti-parallel) 2 -cable of the Hopf link with zero framing. It thus bounds a surface consisting of two annuli. According to the proposition, $L$ does not bound a surface $S$ with $\chi(S)=3$ or $\chi(S)=4$. 


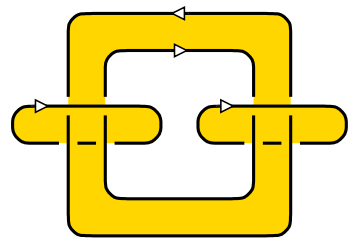

(a) a surface with $\chi=2$

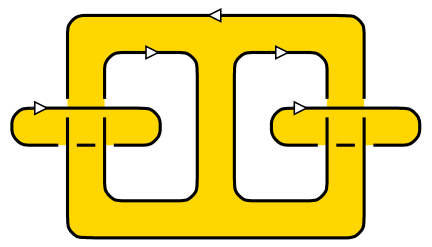

(b) a surface with $\chi=1$

Figure 4: Examples of ribbon surfaces

Example 4.3 The surface $S$ of Figure $4 \mathrm{~b}$ has Euler characteristic $\chi(S)=1+1-1=1$. The link $L=\partial S$ is the connected sum $H_{+} \sharp H_{-} \sharp H_{+} \sharp H_{-}$of four Hopf links, whence

$$
V(L)=\left(q^{+1}+q^{+5}\right)^{2} \cdot\left(q^{-1}+q^{-5}\right)^{2} .
$$

We thus find $\operatorname{det}(L)=16$ and null $V(L)=0$. This example shows that the lower bound for null $V(L)$ does not only depend on the number of disk components of $S$.

Proof of Proposition 4.1 We proceed by induction on the ribbon number $r(S)$ of the ribbon surface $S$, that is, the number of ribbon singularities. If $r(S)=0$, then $S=S_{0} \sqcup \bigcirc^{n}$, and so $V(L)$ is divisible by $V\left(\bigcirc^{n}\right)$. To see this, notice that a connected surfaces with positive Euler characteristic is either a sphere, a projective plane, or a disk. Since $S$ has no closed components, this implies that $\chi(S)=n>0$ can only be realized by (at least) $n$ disks. If the immersed surface $S$ has no singularities, then it is in fact embedded in $\mathbb{R}^{3}$ and so $L$ has (at least) $n$ trivial components.

For the induction step we assume that $r(S) \geq 1$ and that the assertion is true for all ribbon surfaces $S^{\prime}$ with $r\left(S^{\prime}\right)<r(S)$. We replace one ribbon singularity by a band crossing, that is,

$$
\text { we transform } \quad S=\frac{--\mid-}{\square-} \text { into } \quad S^{\prime}=\frac{\frac{1}{1+}}{\frac{1}{1+}} \text {. }
$$

For $L^{\prime}=\partial S^{\prime}$ we know by induction that $V\left(L^{\prime}\right)$ is divisible by $V\left(\bigcirc^{n}\right)$.

We represent the link $L$ and its ribbon surface $S$ by a band diagram $D$. Since the Jones polynomial $V(L)$ and the bracket polynomial $\langle D\rangle$ satisfy $V(L)= \pm A^{k}\langle D\rangle$ for some exponent $k \in \mathbb{Z}$, the assertion for the Jones polynomial $V(L)$ and the bracket polynomial $\langle D\rangle$ are equivalent. In the rest of the proof we will work with the latter. 
Subtracting Equations (4) and (5) we obtain the following difference:

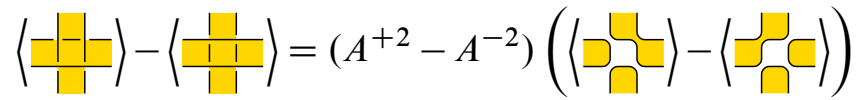

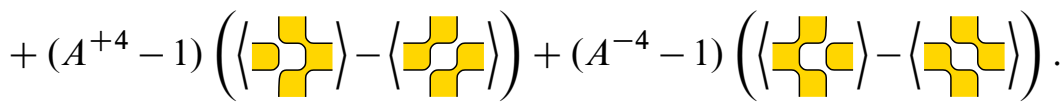

All ribbon surfaces on the right hand side have ribbon number smaller than $r(S)$, so we can apply our induction hypothesis. Cutting open a band increases the Euler characteristic by one, whereas regluing decreases the Euler characteristic by one:

$$
\chi(\supset \square)=\chi(\square)+1 \text {. }
$$

On the right hand side of Equation (7), the two surfaces in the first parenthesis have Euler characteristic $n+1$, the four surfaces in the second parenthesis have Euler characteristic $n$, so each bracket polynomial on the right hand side is divisible by $\left\langle\bigcirc^{n}\right\rangle$. We conclude that $\langle D\rangle$ is divisible by $\left\langle\bigcirc^{n}\right\rangle$ and this completes the proof by induction.

\section{Band crossing changes}

Proposition 4.1 says that $V(L)$ is divisible by $V\left(\bigcirc^{n}\right)=\left(q^{+}+q^{-}\right)^{n-1}$ whenever $L$ bounds a ribbon surface $S$ of positive Euler characteristic $n$. The value $[L / S]:=$ $\left[V(L) /\left(q^{+}+q^{-}\right)^{n-1}\right]_{(q \mapsto i)}$ is thus well-defined (Section 5.1) and we show that it is invariant under certain operations on the surface $S$, namely band crossing changes (Section 5.2) and band twists (Section 5.3). We generalize these observations and establish a convenient framework by introducing the notion of surface invariants of finite type with respect to band crossing changes (Section 5.5).

\subsection{The surface determinant}

We fix the following notation.

Definition 5.1 (surface determinant) Consider an oriented link $L \subset \mathbb{R}^{3}$ bounding an immersed ribbon surface $S \subset \mathbb{R}^{3}$ with Euler characteristic $n=\chi(S)$. We define the determinant of the pair $(L, S)$ to be $[L / S]:=\left[V(L) /\left(q^{+}+q^{-}\right)^{n-1}\right]_{q \mapsto i}$.

Remark 5.2 For $n=1$ this is the ordinary determinant, $\operatorname{det}(L)=V(L)_{q \mapsto i}$. For $n \leq 0$ we multiply by $\left(q^{+}+q^{-}\right)^{1-n}$ and evaluation at $q=i$ thus yields $[L / S]=0$. 
We do not assume $S$ to be oriented, nor even orientable. In order to speak of the Jones polynomial $V(L)$, however, we have to choose an orientation for the link $L=\partial S$.

In general $[L / S]$ is not determined by the surface $S$ alone: although the unoriented link $L$ is determined by $S$, the orientation of $L$ adds an extra bit of information.

Likewise, $[L / S]$ is not an invariant of the link $L$ alone: different surfaces may have different Euler characteristics, and we do not require $\chi(S)$ to be maximal.

According to Proposition 4.1 the surface determinant $[L / S]$ is non zero only if $\chi(S) \geq 1$ and $S$ maximizes the Euler characteristic of surfaces spanning $L$.

Remark 5.3 Changing the orientation of any link component changes the writhe by a multiple of 4 and thus $[L / S]$ changes by a factor \pm 1 .

If we choose a surface component of $S$ and reverse the orientation of its entire boundary, then the writhe changes by a multiple of 8 , and so $[L / S]$ remains unchanged.

This applies in particular to reversing a link component that bounds a disk or Möbius band. If there are no other components, then $[L / S]$ is independent of orientations.

Example 5.4 We always have $[L / S] \in \mathbb{Z}$ or $[L / S] \in i \mathbb{Z}$, depending on whether $c(L)-\chi(S)$ is even or odd. Here are two simple examples:

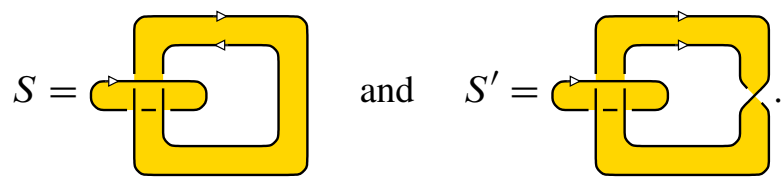

We have $[L / S]=\operatorname{det} L=4$ and $\left[L^{\prime} / S^{\prime}\right]=\operatorname{det} L^{\prime}=-4 i$. We remark that $L=H_{-} \sharp H_{+}$, and we can change orientations so as to obtain $H_{-} \sharp H_{-}$or $H_{+} \sharp H_{+}$, both with determinant -4 . Notice that $\left[L^{\prime} / S^{\prime}\right]$ is independent of orientations.

\subsection{Band crossing changes}

The following observation will be useful.

Proposition 5.5 The surface determinant $[L / S]$ is invariant under band crossing changes.

Proof Let $S$ be an immersed ribbon surface of positive Euler characteristic $n=\chi(S)$. We reconsider Equation (5), resolving a band crossing according to Kauffman's skein relation. The resolution for the changed band crossing is analogous, with all diagrams 
rotated by $90^{\circ}$. When we calculate their difference, 10 of the 16 terms cancel each other in pairs, and we obtain the following skein relation:

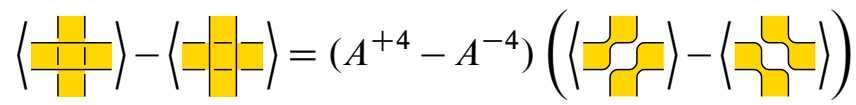

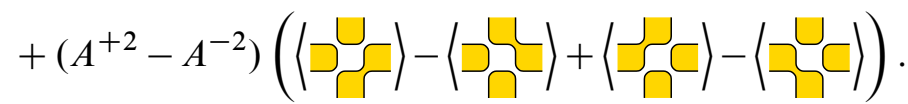

The two surfaces in the first parenthesis have Euler characteristic $n$, so their polynomials are divisible by $\left\langle\bigcirc^{\eta}\right\rangle=\left(q^{+}+q^{-}\right)^{n-1}$, and the coefficient $q^{-2}-q^{+2}=\left(q^{-}+q^{+}\right)\left(q^{-}-q^{+}\right)$ contributes another factor. The four surfaces in the second parenthesis have Euler characteristic $n+1$, so their polynomials are divisible by $\left\langle\bigcirc^{n+1}\right\rangle=\left(q^{+}+q^{-}\right)^{n}$.

This means that $\langle D\rangle$ modulo $\left(q^{+}+q^{-}\right)^{n}$ is invariant under band crossing changes as stated. The writhe remains constant or changes by \pm 8 . We conclude that the Jones polynomial $V(L)$ modulo $\left(q^{+}+q^{-}\right)^{n}$ is invariant under band crossing changes. $\square$

\subsection{Band twists}

Generalizing Example 5.4, we obtain the following result.

Proposition 5.6 The surface determinant $[L / S]$ is invariant under band twisting, up to some sign factor $\varepsilon \in\{ \pm 1, \pm i\}$. More precisely, we have

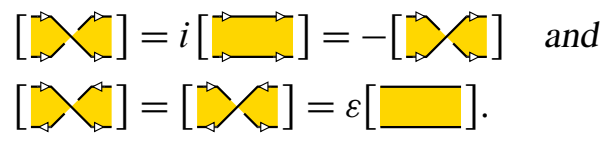

For the last link we have to choose arbitrary orientations; there is no canonical choice.

Proof We have $\langle\supset\rangle=A\langle\bar{\square}\rangle+A^{-1}\langle\supset \square\rangle$. The last term does not contribute to the surface determinant because it has greater Euler characteristic. The other two terms establish the desired equality upon normalization with respect to the writhe. For parallel orientations we obtain:

$$
\begin{aligned}
& {\left[\triangle \searrow_{\triangleright}^{-\infty}=\left[\left(-A^{-3}\right)^{w+1}\langle\supset\rangle /\left(q^{+}+q^{-}\right)^{\chi-1}\right]_{(q \mapsto i)}\right.} \\
& =\left[-A^{-2}\right]_{(q \mapsto i)} \cdot\left[\left(-A^{-3}\right)^{w}\langle\square\rangle /\left(q^{+}+q^{-}\right)^{\chi-1}\right]_{(q \mapsto i)}=i[\stackrel{\longrightarrow}{\longrightarrow}] .
\end{aligned}
$$

We recall our sign convention $q=-A^{-2}$. For anti-parallel orientations we obtain:

$$
\begin{aligned}
& {[\curvearrowright]=\left[\left(-A^{-3}\right)^{w-1}\langle\supset\rangle /\left(q^{+}+q^{-}\right)^{\chi-1}\right]_{(q \mapsto i)}} \\
& =\left[-A^{4}\right]_{(q \mapsto i)} \cdot\left[\left(-A^{-3}\right)^{w}\langle\square\rangle /\left(q^{+}+q^{-}\right)^{\chi-1}\right]_{(q \mapsto i)} \\
& =\left[A^{8}\right]_{(q \mapsto i)} \cdot\left[\left(-A^{-3}\right)^{w+1}\langle\supset\rangle /\left(q^{+}+q^{-}\right)^{\chi-1}\right]_{(q \mapsto i)}=[\precsim] \text {. }
\end{aligned}
$$


The middle term can be identified with $\pm[\overleftrightarrow{\hookrightarrow \triangleleft}]$ or $\pm i[\stackrel{\hookrightarrow \hookrightarrow}{\hookrightarrow}]$. In general the sign depends on the chosen orientations and the induced writhe of the resulting diagram.

Remark 5.7 Adding two full band twists leaves $[L / S]$ invariant. Of course, this can also be realized by a band crossing change, as in the following example:

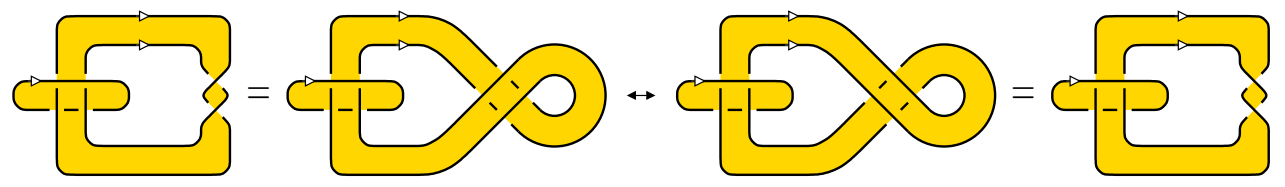

Example 5.8 As a word of warning, the following example illustrates that band twisting or a band crossing change can alter the determinant of a link. We consider

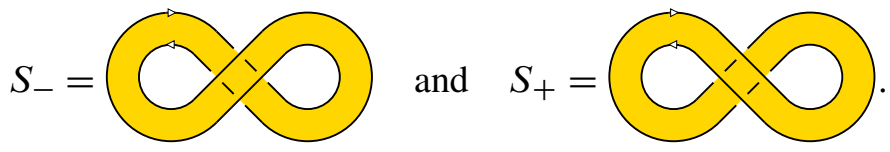

The boundary $H_{ \pm}=\partial S_{ \pm}$is the Hopf link with linking number $\operatorname{lk}\left(H_{ \pm}\right)= \pm 1$ and determinant det $H_{ \pm}= \pm 2 i$. Proposition 5.5 and Proposition 5.6 apply to the surface determinant $\left[L_{ \pm} / S_{ \pm}\right]$, but in this case the statement is empty because $\left[L_{ \pm} / S_{ \pm}\right]=0$. See Remark 5.2.

\subsection{Orientable surfaces}

In order to simplify the exposition we will concentrate on orientable surfaces. This restriction seems acceptable because we are ultimately interested in ribbon links. All results extend to non-orientable surfaces as well, but statements and proofs are twice as long due to clumsy case distinctions.

Definition 5.9 If $S$ is orientable then we define $[S]:=[L / S]$ by choosing an arbitrary orientation of $S$ and the induced orientation of the boundary $L=\partial S$. This is welldefined according to Remark 5.3.

Proposition 5.10 For every orientable surface $S$ we have $[S] \in \mathbb{Z}$.

Proof We have $\chi(S) \equiv c(L) \bmod 2$, where $c(L)$ is the number of components of the link $L=\partial S$. The Jones polynomial $V(L)$ is even if $c(L)$ is odd, and $V(L)$ is odd if $c(L)$ is even. The reduced polynomial $V(L) /\left(q^{+}+q^{-}\right)^{\chi-1} \in \mathbb{Z}\left[q^{ \pm}\right]$is always even. 
Remark 5.11 By definition, $[S]$ depends only on the link $L=\partial S$ and the Euler characteristic $\chi(S)$ of the surface $S$. According to Proposition 5.5 and Proposition 5.6 , the value $[S]$ does not depend on the situation of $S$ in $\mathbb{R}^{3}$, but only on the abstract surface together with the combinatorial pattern of ribbon singularities. This is rather surprising.

\subsection{Surface invariants of finite type}

In order to put the surface determinant into a wider perspective, I would like to expound an interesting analogy with link invariants of finite type. A more comprehensive study of surface invariants of finite type will be the object of a forthcoming article [10].

Remark 5.12 We expand the Jones polynomial $V(q)=\sum_{k=0}^{\infty} v_{k} h^{k}$ in $q=\exp (h / 2)$. Here any power series $q \equiv 1+h / 2 \bmod h^{2}$ could be used: the crucial point is that $q-q^{-1} \equiv h$ has no constant term. Then the link invariants $L \mapsto v_{k}(L)$ are of finite type in the sense of Vassiliev [46] and Goussarov [19], see also Birman-Lin [4] and Bar-Natan [2]. This means that these invariants behave polynomially with respect to crossing changes $\backslash \leftrightarrow \pitchfork$.

Remark 5.13 We can also expand $V(q)=\sum_{k=0}^{\infty} d_{k} h^{k}$ in $q=i \exp (h / 2)$. Any power series $q \equiv i+i h / 2 \bmod h^{2}$ could be used: the crucial point is that $q+q^{-1} \equiv i h$ has no constant term. We obtain a family of link invariants $L \mapsto d_{k}(L)$ starting with the classical link determinant $d_{0}(L)=\left.V(L)\right|_{(q \mapsto i)}=\operatorname{det}(L)$. The Jones nullity $v=$ null $V(L)$ is the smallest index such that $d_{v}(L) \neq 0$. If $L$ bounds a surface $S$ of positive Euler characteristic $n$, then $d_{0}(L)=\cdots=d_{n-2}(L)=0$ and $d_{n-1}(L)=$ $i^{n-1}[L / S]$.

The arguments used in the proofs of Proposition 5.5 and Proposition 5.6 motivate the following definition of alternating sums of surfaces, imitating finite type invariants of links.

Notation As in Section 3.1 we consider a smooth compact surface $\Sigma$ without closed components. In order to simplify we assume $\Sigma$ to be oriented and endow $\partial \Sigma$ with the induced orientation. We denote by $\mathfrak{B}(\Sigma)$ the set of band immersions $\Sigma \uparrow \rightarrow \mathbb{R}^{3}$ modulo ambient isotopy.

Let $D$ be a band diagram representing some ribbon surface $S \in \mathfrak{B}(\Sigma)$ and let $X$ be a set of band crossings of $D$. For each subset $Y \subset X$ we denote by $D_{Y}$ the diagram obtained from $D$ by changing all band crossings $x \in Y$ as indicated in Figure 5a. This 


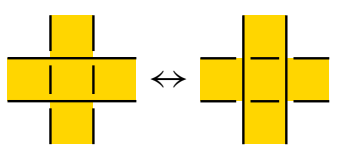

(a) Band crossing

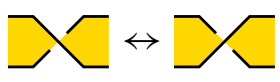

(b) Band twist

Figure 5: Changing band crossings and band twists

does not change the abstract surface $\Sigma$, and so the diagram $D_{Y}$ represents again a ribbon surface in $\mathfrak{B}(\Sigma)$.

Slightly more generally, we also allow $X$ to contain band twists, in which case we simply change one crossing as in Figure $5 \mathrm{~b}$. We will usually not mention this explicitly but rather subsume it under the notion of band crossing change. Of course, two full band twist can be traded for one band crossing change, see Remark 5.7.

Remark 5.14 We emphasize that we are considering links $L$ equipped with extra structure, namely the given surface $S \subset \mathbb{R}^{3}$ spanning $L=\partial S$. This extra structure is crucial. Kauffman [22, chapter V] studied pass moves, which consist of the move of Figure 5a without keeping track of surfaces. He shows that the set of knots splits into two equivalence classes, corresponding to the two values of the Arf invariant.

Remark 5.15 We assume that $\Sigma$ is a compact surface without closed components. Then any two embeddings $f, g: \Sigma \hookrightarrow \mathbb{R}^{3}$ can be transformed one into the other by a finite sequence of the above band crossing changes. The same holds true for ribbon immersions $f, g: \Sigma \uparrow \mathbb{R}^{3}$ provided that the combinatorial structure of their singularities coincide.

Definition 5.16 Let $v: \mathfrak{B}(\Sigma) \rightarrow A$ be a surface invariant with values in some abelian group $A$. We say that $v$ is of degree $\leq m$ with respect to band crossing changes if

$$
\sum_{Y \subset X}(-1)^{|Y|} v\left(D_{Y}\right)=0 \text { whenever }|X|>m .
$$

We say that $v$ is a surface invariant of finite type if it is of degree $\leq m$ for some $m \in \mathbb{N}$.

Remark 5.17 If $A$ is a module over a ring $\mathbb{K}$, then the surface invariants $\mathfrak{B}(\Sigma) \rightarrow A$ of degree $\leq m$ form a module over $\mathbb{K}$. If $A$ is an algebra over $\mathbb{K}$, then the surface invariants $\mathfrak{B}(\Sigma) \rightarrow A$ of finite type form a filtered algebra over $\mathbb{K}$ : if $f$ is of degree $\leq m$ and $g$ is of degree $\leq n$, then their product $f \cdot g$ is of degree $\leq m+n$.

Proposition 5.18 The surface invariant $S \mapsto d_{k}(\partial S)$ is of finite type with respect to band crossing changes. More precisely, it is of degree $\leq m$ for $m=k+1-\chi(S)$. 
In the case $m<0$ we have $k<\chi(S)-1$, whence $d_{k}(\partial S)=0$ by Proposition 4.1. For $m=0$ we have $k=\chi(S)-1$, whence $d_{k}(\partial S)$ is invariant under band crossing changes by Proposition 5.5. Proposition 5.18 extends these results in a natural way to all $k \in \mathbb{N}$. It is a consequence of the following observation.

Lemma 5.19 Consider an oriented band diagram $D$. For every set $X$ of band crossings, the polynomial $\sum_{Y \subset X}(-1)^{|Y|} V\left(\partial D_{Y}\right)$ is divisible by $\left(q^{+}+q^{-}\right)^{|X|+\chi(S)-1}$.

Proof We proceed by induction on the cardinality of $X$. The case $|X|=0$ is settled by Proposition 4.1. If $|X| \geq 1$ then we choose one band crossing or band twist $x \in X$. In the first case we apply Equation (9). The orientations of the vertical and horizontal strands are antiparallel, so we can put them into the following configuration:

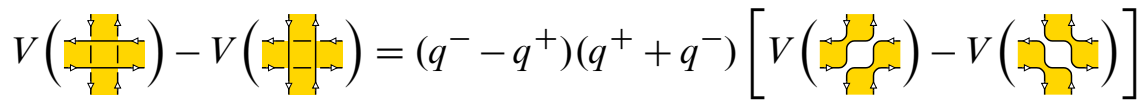

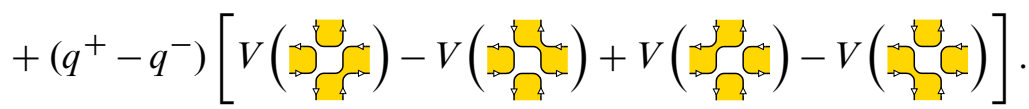

The diagrams so obtained have the same writhe, and thus Equation (7) for the Kauffman bracket directly translates to Equation (10) for the Jones polynomial. On the right hand side the first two terms have the same Euler characteristic as $S$ but one extra factor $\left(q^{+}+q^{-}\right)$, whereas in the last four terms the Euler characteristic increases by one.

The second case is analogous: if $x$ is a band twist, then Equation (3) yields

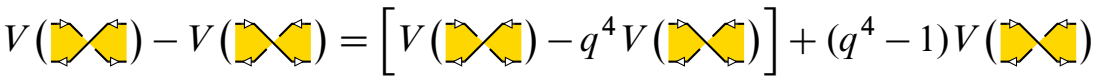

$$
\begin{aligned}
& =\left(q^{1}-q^{3}\right) V\left(\bowtie \varsigma_{\varpi}^{-}\right)+\left(q^{3}-q^{1}\right)\left(q^{+}+q^{-}\right) V(\searrow)
\end{aligned}
$$

In both cases we pass to the alternating sum over all subsets $Y$ of $X^{\prime}=X \backslash\{x\}$. On the left hand side we obtain the alternating sum over all subsets of $X$, as desired. On the right hand side we apply the induction hypothesis to conclude that the resulting polynomial is divisible by $\left(q^{+}+q^{-}\right)^{|X|+\chi(S)-1}$.

Remark 5.20 Every link invariant $L \mapsto v(L)$ of degree $\leq m$ (with respect to crossing changes) induces a surface invariant $S \mapsto v(\partial S)$ of degree $\leq m$ (with respect to band crossing changes). This holds, for example, for the coefficients $v_{k}$ in the above expansion $V(q)=\sum_{k=0}^{\infty} v_{k} h^{k}$ in $q=\exp (h / 2)$. It is surprising that the expansion $V(q)=\sum_{k=0}^{\infty} d_{k} h^{k}$ in $q=i \exp (h / 2)$ provides an independent family of examples, even though the link invariants $d_{k}$ are not of finite type with respect to crossing changes. 
The determinant $d_{0}(L)=\operatorname{det}(L)$ comes close to being a Vassiliev-Goussarov invariant in the sense that $\operatorname{det}(L)^{2}$ is polynomial of degree $\leq 2$ on every twist sequence, see Eisermann [9, Section 5]. Here $\operatorname{det}(\partial S)$ turns out to be of degree $\leq 1-\chi(S)$ with respect to band crossing changes of the surface $S$.

Remark 5.21 We parameterize the Alexander-Conway polynomial $\Delta(L)=\sum a_{k}(L) z^{k}$ by $z=q^{+}-q^{-}$. The link invariant $L \mapsto a_{k}(L)$ is then of degree $k$ with respect to crossing changes. If we consider a disk $\Sigma$ and band immersions $\Sigma \rightarrow \mathbb{R}^{3}$, then the surface invariant $S \mapsto a_{k}(\partial S)$ is of degree 0 with respect to band crossing changes. To see this, notice that the Seifert matrix of the knot $K=\partial S$ has the form $\theta=\left(\begin{array}{cc}0 & A \\ B & C\end{array}\right)$, see Kauffman [22, chapter VIII]. This implies that $\operatorname{sign}(K)=\operatorname{sign}\left(\theta+\theta^{*}\right)$ vanishes and that $\Delta(K)=\operatorname{det}\left(q^{-} \theta^{*}-q^{+} \theta\right)$ is of the form $f\left(q^{+}\right) \cdot f\left(q^{-}\right)$with $f \in \mathbb{Z}\left[q^{ \pm}\right]$. Band crossing changes of $S$ only affect the submatrix $C$, and so $\Delta(K)$ remains unchanged. If we pass from the special case of a disk to immersions or embeddings of an arbitrary surface $\Sigma$, then the surface invariant $S \mapsto a_{k}(\partial S)$ is no longer invariant under band crossing changes. Example 5.8 illustrates this for the linking number $a_{1}=1 \mathrm{k}$ when $\Sigma$ is an annulus.

\section{The Jones determinant of ribbon links}

The surface determinant $[S]$ is invariant under band crossing changes, but in general it changes when we replace a ribbon singularity by a band crossing. In order to analyze this in more detail, we spell out an oriented skein relation (Section 6.1) and establish some useful congruences (Section 6.2). We then apply them to ribbon links (Section 6.3) and prove Theorem 2 stated in the introduction. The arguments remain elementary but get increasingly complicated, because our combinatorial approach entails numerous case distinctions. Finally we sketch an application to satellites of ribbon knots (Section 6.4).

\subsection{An oriented skein relation}

We wish to set up a suitable skein relation for the determinant $[S]$ of an orientable ribbon surface $S$. Replacing a ribbon singularity by a band crossing as in Equation (7), we obtain a ribbon surface $S^{\prime}$ with one less singularity. The right hand side of (7) features six diagrams: the first two of these terms vanish at $q=i$ because they have greater Euler characteristic. Hence Equation (7) becomes

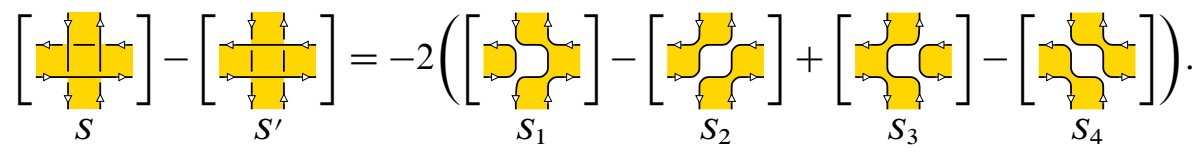


Notice that the orientations of the vertical and horizontal strands are antiparallel, and the writhe of the shown crossings in $S$ and $S^{\prime}$ add up to 0 . Inserting pairs of opposite twists as necessary, we can always put the bands into the configuration shown in (12). This has the advantage that we can use the same orientations on the right hand side. All diagrams have the same writhe, so that Equation (7) for the Kauffman bracket directly translates to the Jones polynomial, and to Equation (12) for the surface determinant.

\subsection{Some useful congruences}

We continue to consider an orientable ribbon surface $S$. We denote by $c(S)$ the number of its connected components. Since each component has Euler characteristic $\leq 1$, the deficiency $d(S)=c(S)-\chi(S)$ is non-negative, and we have $d(S)=0$ if and only if $S$ consists only of disks. In the following induction the deficiency $d(S)$ measures how far $S$ is from being a collection of disks.

Definition 6.1 We call a ribbon singularity essential if the pierced component is a disk and the piercing component remains connected after cutting it open along the singularity. We denote by $e(S)$ the number of essential singularities of $S$.

Lemma 6.2 Every oriented ribbon surface $S \subset \mathbb{R}^{3}$ satisfies the following congruences:

(1) If $d(S)=0$, then $[S] \equiv 1 \bmod 8$.

(2) If $d(S)=1$, then $[S] \equiv 4 e(S) \bmod 8$.

(3) If $d(S) \geq 2$, then $[S] \equiv 0 \bmod 2^{d+1}$.

Remark 6.3 The ribbon condition improves the usual congruences by a factor 2 : in general we only have det $K \equiv 1 \bmod 4$ for a knot and det $L \equiv 0 \bmod 2$ for a link.

Case (1) includes the well-known fact that every ribbon knot $K$ satisfies $\operatorname{det}(K) \equiv 1$ mod 8 . This classical result is reproved in our more general setting for ribbon links.

Case (2) could be reduced to $[S] \equiv 0 \bmod 4$, but the refinement modulo 8 will prove indispensable in order to establish Theorem 2 (see Theorem 6.8 below).

Case (3) could likewise be strengthened, but we content ourselves with a weaker formulation that suffices for the inductive proof of Lemma 6.2.

Proof of Lemma 6.2 We first recall that we assume the surface $S$ to be non-empty and without closed components. We also remark that the case $\chi(S) \leq 0$ is trivial, because $d(S) \geq 1$ and $[S]=0$ by definition. In the sequel we can thus assume $\chi(S) \geq 1$. 
We proceed by induction on the number $r(S)$ of ribbon singularities. Suppose first that $r(S)=0$. If $d(S)=0$, then $S=\bigcirc^{\chi}$ with $\chi=\chi(S)$, whence [S] $=1$. If $d(S) \geq 1$ then $S=S_{0} \sqcup \bigcirc^{\chi}$ with $S_{0} \neq \varnothing$, whence $V(L)=\left(q^{+}+q^{-}\right)^{\chi} V\left(L_{0}\right)$ and $[S]=0$.

For the induction step we suppose that $r(S) \geq 1$ and that the statement is true for all surfaces $S^{\prime}$ with $r\left(S^{\prime}\right)<r(S)$. We then replace a ribbon singularity of $S$ by a band crossing as in Equation (12). By our induction hypothesis, we can apply the congruences stated above to the surface $S^{\prime}, S_{1}, S_{2}, S_{3}, S_{4}$. All surfaces have the same Euler characteristic as $S$ but the number of components may differ: we have $c(S)=c\left(S^{\prime}\right)$ and $c\left(S_{i}\right)-c(S) \in\{1,0,-1\}$.

We denote by $S_{=}$resp. $S_{\|}$the component the surface $S$ containing the horizontal resp. vertical strip in Equation (12). In order to analyze the contribution of the four ribbon surfaces $S_{1}, S_{2}, S_{3}, S_{4}$ we distinguish the following cases.

Case (1) If $d(S)=0$, then we are dealing exclusively with disks:

(a) If $S_{=}$and $S_{\|}$are different disks of $S$, then all four diagrams $S_{1}, S_{2}, S_{3}, S_{4}$ feature only disks, whence $d\left(S_{1}\right)=d\left(S_{2}\right)=d\left(S_{3}\right)=d\left(S_{4}\right)=0$. We thus have $\left[S_{1}\right] \equiv\left[S_{2}\right] \equiv\left[S_{3}\right] \equiv\left[S_{4}\right] \equiv 1 \bmod 8$, whence $\left[S_{1}\right]-\left[S_{2}\right]+\left[S_{3}\right]-\left[S_{4}\right] \equiv 0$ $\bmod 8$. The factor -2 in Equation (12) ensures that $[S] \equiv\left[S^{\prime}\right] \bmod 16$.

(b) Suppose next that $S_{\|}$coincides with $S_{=}$. For concreteness we will assume that the western and southern pieces are connected outside of the local picture, as indicated in Figure 6. (The other three variants are analogous.)
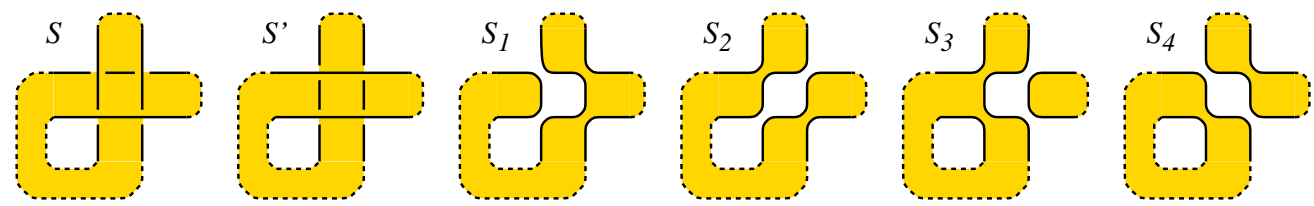

Figure 6: Resolving a pure ribbon singularity

Two diagrams, in our case $S_{1}$ and $S_{2}$, feature only disks, whence $d\left(S_{1}\right)=$ $d\left(S_{2}\right)=0$ and $\left[S_{1}\right] \equiv\left[S_{2}\right] \equiv 1 \bmod 8$. The other two diagrams, in our case $S_{3}$ and $S_{4}$, each feature one extra annulus, whence $d\left(S_{3}\right)=d\left(S_{4}\right)=1$, whence $\left[S_{3}\right] \equiv\left[S_{4}\right] \equiv 0 \bmod 4$. Equation (12) implies that $[S] \equiv\left[S^{\prime}\right] \bmod 8$.

Remark 6.4 For future reference, we wish to be more precise here. The surfaces $S_{3}$ and $S_{4}$ may have different numbers of essential singularities, so $\left[S_{4}\right]-\left[S_{3}\right]=4 \Delta$ mod 8 . We conclude that $[S]-\left[S^{\prime}\right] \equiv 8 \Delta \bmod 16:$ if $\Delta$ is even, then $[S] \equiv\left[S^{\prime}\right]$ $\bmod 16$; if $\Delta$ is odd, then $[S]-\left[S^{\prime}\right] \equiv 8 \bmod 16$. 
The difference $\Delta=e\left(S_{4}\right)-e\left(S_{3}\right)$ is the number of times that the annulus formed by connecting the western and southern pieces, essentially pierces the northern piece: these singularities are essential for $S_{4}$ but not essential for $S_{3}$. All other essential singularities are the same for both $S_{3}$ and $S_{4}$.

Case (2) If $d(S)=1$, then we are dealing with $n$ disks and one annulus.

(a) If the components $S_{=}$and $S_{\|}$coincide, then $d\left(S_{1}\right)=d\left(S_{2}\right)=d\left(S_{3}\right)=d\left(S_{4}\right) \geq$ 1 , whence $\left[S_{1}\right] \equiv\left[S_{2}\right] \equiv\left[S_{3}\right] \equiv\left[S_{4}\right] \equiv 0 \bmod 4$. In this case $[S] \equiv\left[S^{\prime}\right] \bmod 8$. The considered singularity is not essential, so that $e(S)=e\left(S^{\prime}\right)$.

In the following cases we assume that $S_{=}$and $S_{\|}$are different components.

(b) If splitting separates both $S_{=}$and $S_{\|}$, then $d\left(S_{1}\right)=d\left(S_{2}\right)=d\left(S_{3}\right)=d\left(S_{4}\right)=1$, whence $\left[S_{1}\right] \equiv\left[S_{2}\right] \equiv\left[S_{3}\right] \equiv\left[S_{4}\right] \equiv 0 \bmod 4$. We conclude that $[S] \equiv\left[S^{\prime}\right]$ mod 8 . The considered singularity is not essential, so that $e(S)=e\left(S^{\prime}\right)$.

(c) If splitting separates $S_{\|}$but not $S_{=}$, then $d\left(S_{1}\right)=d\left(S_{2}\right)=d\left(S_{3}\right)=d\left(S_{4}\right)=0$, whence $\left[S_{1}\right] \equiv\left[S_{2}\right] \equiv\left[S_{3}\right] \equiv\left[S_{4}\right] \equiv 1 \bmod 8$. We conclude that $[S] \equiv\left[S^{\prime}\right]$ mod 16. The considered singularity is not essential, so that $e(S)=e\left(S^{\prime}\right)$.

(d) If splitting separates $S_{=}$but not $S_{\|}$, then $d\left(S_{1}\right)=d\left(S_{3}\right)=1$ and $d\left(S_{2}\right)=$ $d\left(S_{4}\right)=0$. We thus have $\left[S_{1}\right] \equiv\left[S_{3}\right] \equiv 0 \bmod 4$ as well as $\left[S_{2}\right] \equiv\left[S_{4}\right] \equiv 1$ $\bmod 8$, whence $\left[S_{1}\right]-\left[S_{2}\right]+\left[S_{3}\right]-\left[S_{4}\right] \equiv 2 \bmod 4$. We conclude that $[S] \equiv$ $\left[S^{\prime}\right]+4 \bmod 8$. The considered singularity is essential, so that $e(S)=e\left(S^{\prime}\right)+1$.

This exhausts all possibilities in the case $d(S)=1$ : at least one of the components $S_{=}$or $S_{\|}$is a disk, and so splitting separates at least one of them.

Case (3) In the case $d(S)=1$ we already know that $[S] \equiv 0 \bmod 2^{d+1}$. If $d(S) \geq 2$ then the four surfaces $S_{1}, S_{2}, S_{3}, S_{4}$ satisfy $d\left(S_{i}\right) \geq d(S)-1$, whence $\left[S_{i}\right] \equiv 0 \bmod 2^{d}$. Equation (12) then implies that $[S] \equiv\left[S^{\prime}\right] \bmod 2^{d+1}$.

\subsection{Application to ribbon links}

For a ribbon knot $K=\partial S$, Proposition 5.5 says that $\operatorname{det}(K)$ is invariant under band crossing changes of $S$. This is a well-known property for the classical determinant: even the Alexander-Conway polynomial $\Delta(K)$ does not change (see Remark 5.21). This observation trivially holds for ribbon links with $n \geq 2$ components, for which we always have $\Delta(L)=0$. The point of Proposition 5.5 is that after dividing out the factor $V\left(\bigcirc^{n}\right)$ in $V(L)$ we obtain the desired property for the Jones determinant. 
Corollary 6.5 Consider an $n$-component ribbon link $L$ bounding a collection of ribbon disks $S \subset \mathbb{R}^{3}$. Then the Jones nullity is null $V(L)=n-1$, and the determinant $\operatorname{det} V(L)=[S]$ is invariant under band crossing changes.

Proof We know from Corollary 2.6 that null $V(L) \leq n-1$ for all $n$-component links. According to Proposition 4.1 we have null $V(L) \geq n-1$ for $n$-component ribbon links. We conclude that null $V(L)=n-1$ and so $\operatorname{det} V(L)=\left[V(L) / V\left(\bigcirc^{n}\right)\right]_{(q=i)}=[S]$. Proposition 5.5 ensures that det $V(L)$ is invariant under band crossing changes.

Corollary 6.6 Consider an $n$-component ribbon link $L=K_{1} \cup \cdots \cup K_{n}$ that bounds a collection of ribbon disks $S \subset \mathbb{R}^{3}$ without mixed ribbon singularities, which means that distinct disks never intersect each other. Then the Jones determinant satisfies $\operatorname{det} V(L)=\operatorname{det}\left(K_{1}\right) \cdots \operatorname{det}\left(K_{n}\right)$ and is thus a square integer.

Proof Since there are no mixed ribbon singularities, we can change band crossings from $S=S_{1} \cup \cdots \cup S_{n}$ to $S^{\prime}=S_{1} \sqcup \cdots \sqcup S_{n}$. Using the invariance established in Corollary 6.5, we conclude that $\operatorname{det} V(L)=\operatorname{det} V\left(L^{\prime}\right)=\operatorname{det}\left(K_{1}\right) \cdots \operatorname{det}\left(K_{n}\right)$.

Remark 6.7 If we allow ribbon disks to intersect each other, then multiplicativity holds at least modulo 16: for mixed ribbon singularities, the proof of case (1a) of Lemma 6.2 shows that $[S] \equiv\left[S^{\prime}\right]$ mod 16 holds in Equation (12). Having replaced all mixed ribbon singularities by ribbon crossings, we can apply Corollary 6.6 to conclude that $\operatorname{det} V(L) \equiv \operatorname{det} V\left(L^{\prime}\right)=\operatorname{det}\left(K_{1}\right) \cdots \operatorname{det}\left(K_{n}\right)$, so in particular det $V(L) \equiv 1 \bmod 8$. We have to work a bit harder to improve this congruence from 16 to 32 , which is where the full details of Lemma 6.2 come into play.

Theorem 6.8 Consider an $n$-component ribbon link $L=K_{1} \cup \cdots \cup K_{n}$, bounding a collection of ribbon disks $S \subset \mathbb{R}^{3}$. Suppose that in Equation (12) the depicted ribbon singularity involves two distinct disks, $S_{=} \neq S_{\|}$. Then $\left[S_{1}\right]-\left[S_{2}\right] \equiv\left[S_{3}\right]-\left[S_{4}\right]$ $\bmod 16$ and thus $[S] \equiv\left[S^{\prime}\right] \bmod 32$.

Proof We proceed by a double induction. The first induction is on the ribbon number $r(S)$. If $r(S)=1$, then all links are trivial ie $L=L^{\prime}=L_{1}=L_{2}=L_{3}=L_{4}=\bigcirc^{n}$, and so $[S]=\left[S^{\prime}\right]=\left[S_{1}\right]=\left[S_{2}\right]=\left[S_{3}\right]=\left[S_{4}\right]=1$.

If $r(S) \geq 2$, we proceed by induction on the number $k$ of mixed singularities of $S_{=}$. If $k=1$ then $S=$ is not involved in any other mixed ribbon singularity besides the shown one. Applying band crossing changes (Corollary 6.5) we can achieve that $S_{=}$ lies above all other components, except of course at the shown ribbon singularity. This situation is depicted in Figure 7: $S_{1}$ and $S_{3}$ are two connected sums, while $S_{2}$ and 
$S_{4}$ are mutants modulo some band twisting (see Proposition 5.6). This implies that $\left[S_{1}\right]=\left[S_{3}\right]$ and $\left[S_{2}\right]=\left[S_{4}\right]$. The difference $\left[S_{1}\right]-\left[S_{2}\right]=\left[S_{3}\right]-\left[S_{4}\right]$ is a multiple of 8 according to Remark 6.7. Equation (12) then implies that $[S] \equiv\left[S^{\prime}\right] \bmod 32$.
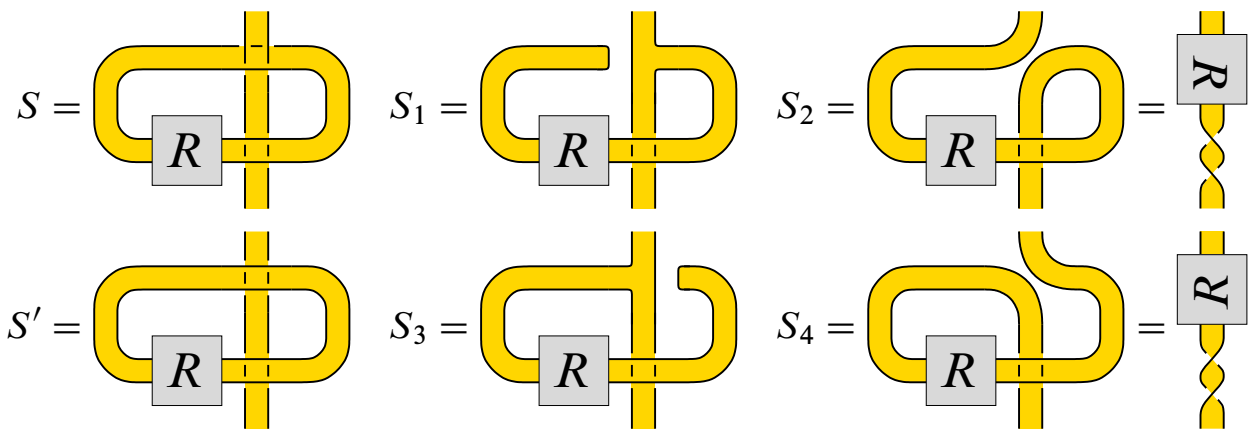

Figure 7: Resolving the only ribbon singularity between $S_{=}$and $S_{\|}$

Suppose next that $k \geq 2$, that is, $S_{=}$is involved in another mixed ribbon singularity. By induction, it suffices to replace one such ribbon singularity by a band crossing. This replacement translates $S, S^{\prime}, S_{1}, S_{2}, S_{3}, S_{4}$ to six new diagrams $\bar{S}, \bar{S}^{\prime}, \bar{S}_{1}, \bar{S}_{2}, \bar{S}_{3}, \bar{S}_{4}$, each having one less ribbon singularity. By induction we know that $[\bar{S}] \equiv\left[\bar{S}^{\prime}\right] \bmod 32$.

If our second ribbon singularity involves $S_{=}$and some third component different from $S_{\|}$, then we can apply Remark 6.7 to all four diagrams on the right hand side to obtain the congruence $\left[S_{1}\right]-\left[\bar{S}_{1}\right] \equiv\left[S_{2}\right]-\left[\bar{S}_{2}\right] \equiv\left[S_{3}\right]-\left[\bar{S}_{3}\right] \equiv\left[S_{4}\right]-\left[\bar{S}_{4}\right] \equiv 0 \bmod 16$. Equation (12) then implies that $[S] \equiv\left[S^{\prime}\right] \bmod 32$.
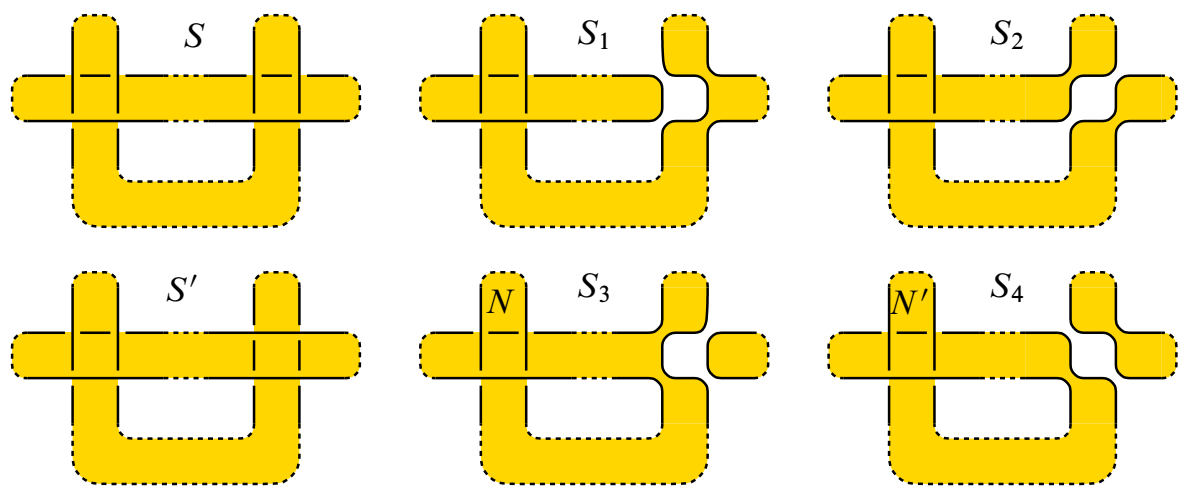

Figure 8: Resolving a second ribbon singularity between $S_{=}$and $S_{\|}$ 
The only problem arises when our second ribbon singularity involves both $S=$ and $S_{\|}$. Suppose for example that the western and southern pieces of $S$ meet again in a second ribbon singularity, as depicted in Figure 8 . Then this is still a mixed singularity in $S_{1}$ and $S_{2}$, and so $\left[S_{1}\right]-\left[\bar{S}_{1}\right] \equiv\left[S_{2}\right]-\left[\bar{S}_{2}\right]=0 \bmod 16$ by Remark 6.7. But in $S_{3}$ and $S_{4}$ it becomes a pure singularity. Remark 6.4 in the proof of Lemma 6.2 case (1b) shows that $\left[S_{3}\right]-\left[\bar{S}_{3}\right] \equiv\left[S_{4}\right]-\left[\bar{S}_{4}\right] \equiv 8 \Delta \bmod 16$ : the northern pieces $N$ and $N^{\prime}$ are pierced by the newly formed annulus in exactly the same essential singularities. We conclude that

$$
\begin{aligned}
{[S]-\left[S^{\prime}\right] } & \equiv 2\left(\left[S_{1}\right]-\left[S_{2}\right]+\left[S_{3}\right]-\left[S_{4}\right]\right) \\
& \equiv 2\left(\left[\bar{S}_{1}\right]-\left[\bar{S}_{2}\right]+\left[\bar{S}_{3}\right]-\left[\bar{S}_{4}\right]\right) \equiv[\bar{S}]-\left[\bar{S}^{\prime}\right] \equiv 0 \bmod 32
\end{aligned}
$$

because $\left[S_{1}\right] \equiv\left[\bar{S}_{1}\right]$ and $\left[S_{2}\right] \equiv\left[\bar{S}_{2}\right]$ and $\left[S_{3}\right]-\left[S_{4}\right] \equiv\left[\bar{S}_{3}\right]-\left[\bar{S}_{4}\right]$ modulo 16 .

Corollary 6.9 (general multiplicativity modulo 32) Every $n$-component ribbon link $L=K_{1} \cup \cdots \cup K_{n}$ satisfies the congruence $\operatorname{det} V(L) \equiv \operatorname{det}\left(K_{1}\right) \cdots \operatorname{det}\left(K_{n}\right) \bmod 32$.

Proof We first replace all mixed ribbon singularities by ribbon crossings: Theorem 6.8 ensures that $\operatorname{det} V(L) \equiv \operatorname{det} V\left(L^{\prime}\right) \bmod 32$. We can then apply Corollary 6.6. $\square$

Example 6.10 The value 32 is the best possible: the 2 -component link $L=10 n 36$ depicted below is ribbon, whence $\operatorname{det}(L)=0$, and its Jones polynomial factors as

$V(L)=\left(q^{+}+q^{-}\right)\left(-q^{+8}+2 q^{+6}-3 q^{+4}+4 q^{+2}-3+4 q^{-2}-3 q^{-4}+2 q^{-6}-q^{-8}\right)$.

Here we find $\operatorname{det} V(L)=-23$ whereas the components satisfy $\operatorname{det}\left(K_{1}\right)=1$ and $\operatorname{det}\left(K_{2}\right)=9$. The congruence $-23 \equiv 9 \bmod 32$ is satisfied, and 32 is optimal.
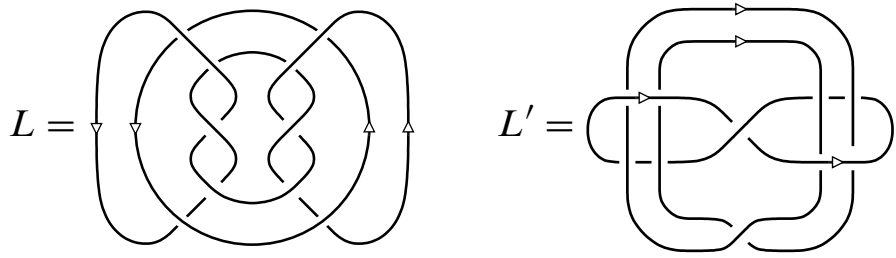

Example 6.11 Not all links with maximal nullity null $V(L)=n-1$ satisfy multiplicativity modulo 32 . For $L^{\prime}=10 n 57$, for example, we find $\operatorname{det}\left(L^{\prime}\right)=0$ and

$$
V\left(L^{\prime}\right)=\left(q^{+}+q^{-}\right)\left(q^{+6}-2 q^{+4}+2 q^{+2}-2+3 q^{-2}-2 q^{-4}+2 q^{-6}-q^{-8}\right)
$$

whence det $V\left(L^{\prime}\right)=-15$. Both components separately are trivial, and det $V\left(L^{\prime}\right) \equiv 1$ holds modulo 16 but not modulo 32. (In particular, $L^{\prime}$ is not a ribbon link. This is no surprise: determinant and signature vanish but the Alexander polynomial does not.) 


\subsection{Satellites of ribbon knots}

Our results contain information for links with two or more components, but at first sight they seem void for knots. One possible application is via the construction of satellites.

Every oriented knot $K \subset \mathbb{R}^{3}$ can be equipped with a tubular neighbourhood, that is, an embedded torus $f: \mathbb{D}^{2} \times \mathbb{S}^{1} \hookrightarrow \mathbb{R}^{3}, f(s, t)=f_{s}(t)$, such that $f_{0}$ parameterizes $K$ satisfying $\operatorname{lk}\left(f_{0}, f_{1}\right)=0$ and $\operatorname{lk}\left(f_{0},\left.f\right|_{\mathbb{S}^{1} \times\{1\}}\right)=+1$. Such an embedding $f$ exists and is unique up to isotopy. For a link $P \subset \mathbb{D}^{2} \times \mathbb{S}^{1}$, the image $f(P) \subset \mathbb{R}^{3}$ is called the satellite of $K$ with pattern $P$, and will be denoted by $K * P$.

Definition 6.12 We say that $P \subset \mathbb{D}^{2} \times \mathbb{S}^{1}$ is a ribbon pattern if $\bigcirc * P$ is a ribbon link, where $\bigcirc$ denotes the trivial knot. This means that the standard (unknotted and untwisted) embedding of the torus $\mathbb{D}^{2} \times \mathbb{S}^{1} \hookrightarrow \mathbb{R}^{3}$ maps $P$ to a ribbon link in $\mathbb{R}^{3}$.

Proposition 6.13 If $K$ is a ribbon knot and $P=P_{1} \cup \cdots \cup P_{n}$ is an $n$-component ribbon pattern, then the satellite $K * P$ is an $n$-component ribbon link.

Remark 6.14 Starting with a ribbon pattern $P$, the satellite $K * P$ may be ribbon even though $K$ is not; see Rolfsen [44, Example 8E33].

Corollary 6.15 If $K$ is a ribbon knot, then for every $c \in \mathbb{N}$ the 0 -framed $c$-cable $K^{c}$ is a ribbon link, whence null $V\left(K^{c}\right)=n-1$ and $\operatorname{det} V\left(K^{c}\right) \equiv \operatorname{det}(K)^{c} \bmod 32$.

Example 6.16 The knot $K=6_{1}$ is the smallest ribbon knot; it has determinant $\operatorname{det}(K)=9$. The Jones determinant of its two-cable is $\operatorname{det} V\left(K^{2}\right)=49=9^{2}-32$. For the three-cable we find $\operatorname{det} V\left(K^{3}\right)=1785=9^{3}+33 \cdot 32$. Again 32 is optimal.

This corollary is quite pleasant, yet it does not seem to obstruct ribbonness. A possible explanation is that every cable $K^{c}$ is a boundary link: Question 7.8 below asks whether this entails the same algebraic consequences, even if the initial knot $K$ is not ribbon.

\section{Open questions and perspectives}

Our results can be seen as a first step towards understanding the Jones polynomial of ribbon links. They suggest further questions and generalizations in several directions. 


\subsection{From ribbon to slice}

At the time of writing it is not known whether every smoothly slice link is a ribbon link. Our results thus offer two perspectives: either they extend from ribbon to smoothly slice links, which would be rather satisfactory for the sake of completeness. Or, even more interestingly, there exist smoothly slice links for which some (suitably refined) ribbon criteria fail: this would refute the long-standing conjecture "smoothly slice implies ribbon" conjecture, at least for links.

Question 7.1 Do Theorem 1 and Theorem 2 generalize from ribbon links to slice links?

Quite possibly our results hold true in this generalized setting. An elegant way to show this would be to extend an observation of Casson, recorded by Livingston [33, Section 2.1]: for every slice knot $K$ there is a ribbon knot $K^{\prime}$ such that their connected sum $K \sharp K^{\prime}$ is ribbon. Is there an analogous trick for slice links?

A negative answer to Question 7.1 would be spectacular, but it remains to be examined whether the Jones polynomial can detect such subtle differences, if at all they exist. As Livingston [33, Section 10, Problem 1] put it: "One has little basis to conjecture here. Perhaps obstructions will arise (...) but the lack of potential examples is discouraging."

\subsection{From Jones to HOMFLYPT}

It is tempting to generalize Theorem 1 to other knot polynomials, in particular to the HOMFLYPT polynomial, or at least to $V_{N}$ for $N$ prime.

Question 7.2 Does Theorem 1 extend to the generalized Jones polynomial in the sense that $V_{N}(L)=V_{N}\left(\bigcirc^{n}\right) \cdot \widetilde{V}_{N}(L)$ for every ribbon link $L$ ?

This holds for $N=0$ because the Alexander-Conway polynomial vanishes for $n \geq 2$. The case $N=1$ is trivial. Theorem 1 settles the case $N=2$. The question for $N \geq 3$ is open, but sample calculations suggest that the factorization seems to hold.

Remark 7.3 The Kauffman bracket has served us well in the inductive proof for $N=2$. For $V_{N}$ with $N \geq 2$, Murakami-Ohtsuki-Yamada [39] have developed an analogous oriented state model. Even though the approach is very similar, the calculations generalizing Section 4 get stuck because certain terms do not cancel each other. This makes the argument harder and some additional ideas will be needed.

Question 7.4 How can Theorem 1 be generalized to the Kauffman polynomial [24]? The obvious generalization is false: the Kauffman polynomial $F(L) \in \mathbb{Z}\left[a^{ \pm}, z^{ \pm}\right]$of the two-component ribbon link $L=10 n 36$, for example, is not divisible by $F\left(\bigcirc^{2}\right)$. 


\subsection{Towards Khovanov homology}

The most fertile development in the geometric understanding and application of the Jones polynomial in recent years has been Khovanov homology [26; 3]. Applying the philosophy of categorification to the Kauffman bracket, this theory associates to each link $L$ a bigraded homology $K h(L)=\bigoplus_{i, j \in \mathbb{Z}} K h_{i, j}(L)$ as an invariant. The polynomial $P(t, q)=\sum_{i, j \in \mathbb{Z}} t^{i} q^{j} \operatorname{dim}_{\mathbb{Q}}\left(K h_{i, j}(L) \otimes \mathbb{Q}\right)$ is an invariant of $L$ that specializes for $t=-1$ to the Jones polynomial, $P(-1, q)=\left(q^{+}+q^{-}\right) \cdot V(L)$.

Question 7.5 What is the homological version of $V(L)=V\left(\bigcirc^{n}\right) \cdot \tilde{V}(L)$ ?

The naïve generalization would be $K h(L) \cong K h\left(\bigcirc^{n}\right) \otimes \widetilde{K} h(L)$. The first problem in stating and proving a result of this type is that the isomorphism must be made explicit and should be as natural as possible. A polynomial factorization such as $P(L)=\left(q^{+}+q^{-}\right)^{n} \cdot \tilde{P}(L)$ is a weaker consequence that does not require isomorphisms in its statement. Sample calculations, say for $L=10 n 36$, show that these simpleminded factorizations do not hold, neither over $\mathbb{Q}$ nor over $\mathbb{Z} / 2$.

Since $P(-1, t)$ can be seen as the graded Euler characteristic of $K h(L)$, another analogy could prove useful: for every fibration $p: E \rightarrow B$ with fibre $F$, the LeraySerre spectral sequence with $E_{p, q}^{2}=H_{p}\left(B, H_{q}(F)\right)$ converges to $H_{p+q}(E)$, whence $\chi(E)=\chi(B) \cdot \chi(F)$. Can the factorization $V(L)=V\left(\bigcirc^{n}\right) \cdot \tilde{V}(L)$ be derived as the Euler characteristic of some spectral sequence? What is the rôle of the factor $\widetilde{V}(L)$ ?

\subsection{Ribbon cobordism}

On top of the quantitative improvement of a more detailed numerical invariant $P(t, q)$, Khovanov homology provides an important qualitative improvement: it is functorial with respect to link cobordism (Jacobsson [20], Khovanov [27]). In this vein Rasmussen [43] established a lower bound for the slice genus of knots, providing a new proof of the Milnor conjecture on the unknotting number of torus knots. It thus seems reasonable to hope that $K h(L)$ captures more subtle properties of slice and ribbon links.

Question 7.6 Is there a functorial version of Theorem 1 and Theorem 2?

Gordon [18] introduced the notion of ribbon concordance. In the slightly more general setting of Proposition 1 we consider a link $L \subset \mathbb{R}^{3}$ that bounds a properly embedded smooth surface $S \subset \mathbb{R}_{+}^{4}$ of positive Euler characteristic $n \geq 1$ and without local minima. Cutting out small disks around $n$ local maxima we obtain a ribbon cobordism $C \subset \mathbb{R}^{3} \times[0,1]$ from $L=C \cap\left(\mathbb{R}^{3} \times\{0\}\right)$ to $\bigcirc^{n}=C \cap\left(\mathbb{R}^{3} \times\{1\}\right)$ such that $\chi(C)=0$. This induces homomorphisms $c: K h(L) \rightarrow K h\left(\bigcirc^{n}\right)$ and $c^{*}: K h\left(\bigcirc^{n}\right) \rightarrow K h(L)$. 
Question 7.7 Is $c$ surjective? Is $c^{*}$ injective? Better still, do we have $c \circ c^{*}=\mathrm{id}$ ? A positive answer would exhibit $K h\left(\bigcirc^{n}\right)$ as a direct summand of $K h(L)$.

C Blanchet suggested that the chain complex $C K h(L)$ could be considered as a module over $C K h\left(\bigcirc^{n}\right)=A^{\otimes n}$, where $A$ is the Frobenius algebra used in Khovanov's construction. This leads to the natural question: when is $C K h(L)$ essentially free over $C K h\left(\bigcirc^{n}\right)$ ? A positive answer would explain the factorization $V(L)=V\left(\bigcirc^{n}\right) \cdot \tilde{V}(L)$ and potentially give some meaning to the reduced Jones polynomial $\tilde{V}(L)$.

\subsection{Other geometric criteria}

We have concentrated here on ribbon links, but many other links $L$ may also satisfy the conclusion of Theorem 1 and Theorem 2:

Question 7.8 Which other geometric properties imply that $V(L)$ is divisible by $V\left(\bigcirc^{n}\right)$ ? Do they imply that $L=K_{1} \cup \cdots \cup K_{n}$ satisfies $\operatorname{det} V(L) \equiv \operatorname{det}\left(K_{1}\right) \cdots \operatorname{det}\left(K_{n}\right)$ modulo 32? More concretely: does this hold for boundary links?

We recall that an $n$-component link $L=L_{1} \cup \cdots \cup L_{n}$ is a boundary link if it bounds a surface $S=S_{1} \cup \cdots \cup S_{n}$ embedded in $\mathbb{R}^{3}$ such that $\partial S_{i}=L_{i}$ for each $i=1, \ldots, n$. (We can always find a connected surface $S$ such that $\partial S=L$, but here we require that $S$ consist of $n$ disjoint surfaces $S_{1}, \ldots, S_{n}$.) The Seifert nullity of a boundary link is maximal, perhaps its Jones nullity too. It is certainly not enough that pairwise linking numbers vanish: the Whitehead link $W$ satisfies $\operatorname{lk}(W)=0$ but $\operatorname{det}(W)=8 i$.

Question 7.9 For which links $L$ do we have equality $\operatorname{null}_{\omega}(L)=\operatorname{null}_{\omega} V_{N}(L)$ ?

The following observations show that this question is not completely absurd:

- Equality holds for all knots $K$ and prime $N$, becausenull $\omega_{\omega}(K)=\operatorname{null}_{\omega} V_{N}(K)=0$, that is, $\operatorname{det}_{\omega}(K)=\left.V_{N}(L)\right|_{(q \mapsto \omega)}$ is always non-zero.

- Equality also holds for all two-component links and prime $N$, because we have $\operatorname{null}_{\omega}(L) \in\{0,1\}$ and $\operatorname{null}_{\omega} V_{N}(L) \in\{0,1\}$, as well as $\operatorname{det}_{\omega}(L)=\left.V_{N}(L)\right|_{(q \mapsto \omega)}$.

- Equality is preserved under disjoint union, connected sum, mirror images, and reversal of orientations.

- Theorem 1 ensures that, at least for $N=2$, equality holds for all ribbon links.

Garoufalidis [17, Corollary 1.5] observed that null $(L) \geq 4$ implies null $V(L) \geq 2$. This follows from Equation (2) and a result of Lescop [30, Section 5.3] saying that $\Lambda(M)$ vanishes for every manifold with $\operatorname{dim} H_{1}(M, \mathbb{Q}) \geq 4$. In the special case $M=\Sigma_{L}^{2}$ this can possibly be sharpened to show that null $(L) \geq 2$ implies null $V(L) \geq 2$. 
Question 7.10 Does link concordance $L \sim L^{\prime}$ imply $\operatorname{null}_{\omega} V_{N}(L)=\operatorname{null}_{\omega} V_{N}\left(L^{\prime}\right)$ ? If so, which congruence holds between $\operatorname{det}_{\omega} V_{N}(L)$ and $\operatorname{det}_{\omega} V_{N}\left(L^{\prime}\right)$ ?

For the Alexander-Conway polynomial the corresponding questions were answered by Kawauchi [25] and Cochran [7]. Equality in Question 7.9 would imply concordance invariance of $\operatorname{null}_{\omega} V_{N}(L)$, because the Seifert nullity is a concordance invariant.

\subsection{Does the Jones polynomial determine the signature mod 4?}

The determinant $\operatorname{det}(L)$ and the signature $\operatorname{sign}(L)$ of a link $L$ are related by the formula

$$
\operatorname{det}(L)=i^{-\operatorname{sign}(L)} \cdot|\operatorname{det}(L)| .
$$

Conway [8] used this together with $\operatorname{sign}\left(\swarrow_{)}\right)-\operatorname{sign}(\searrow) \in\{0,1,2\}$ to calculate signatures recursively. An analogous formula holds for every $\omega \in \mathbb{S}^{1}$ with $\operatorname{im}(\omega)>0$.

If $\omega$ is a primitive $2 N$ th root of unity, we know that $\operatorname{det}_{\omega}(L) \neq 0$ at least for knots. For links with $n \geq 2$ components Conway's signature calculation is obstructed by the fact that the determinant may vanish, in which case Equation (13) contains no information. This happens exactly when null $(L) \geq 1$. One might suspect that the stronger condition $\operatorname{det} V(L)=i^{-\operatorname{sign}(L)} \cdot|\operatorname{det} V(L)|$ holds. Unfortunately this is false in general: see Example 6.10 above for a ribbon link with $\operatorname{det} V(L)<0$.

The formula thus needs some correction. Of course we can define a link invariant $\varepsilon(L): \mathbb{R} \rightarrow\{ \pm 1, \pm i\}$ by $\varepsilon(L):=i^{\operatorname{sign}(L)} \cdot \operatorname{det} V(L) /|\operatorname{det} V(L)|$. The topological meaning of this factor $\varepsilon(L)$, however, is not clear. It is also unknown whether $\varepsilon(L)$ can be deduced from the Jones polynomial alone. If so, then the Jones polynomial would determine the signature of all links via Conway's skein theoretic recursion.

\subsection{Surface invariants of finite type}

Section 5 introduces and illustrates the concept of surface invariants that are of finite type with respect to band crossing changes. This is an interesting analogy and extension of link invariants of finite type. What is the precise relationship between these two classes of invariants? In our examples the surface invariant $S \mapsto d_{k}(\partial S)$ only depends on the boundary of $S$, but in general this need not be the case. Can we generate more non-trivial examples from the HOMFLYPT or the Kauffman polynomial or other quantum invariants? What is their geometric significance?

The general finite type approach to surfaces will be the object of a forthcoming article [10]. Generalizing Section 5, one proceeds as follows. 
- Introduce the filtration induced by band crossing changes and band twists.

- Study the graded quotients and extract combinatorial data modulo relations.

- Integrate (in low degree at least) combinatorial data to invariants of surfaces.

It is interesting to note that the Euler characteristic of the surface intervenes in a natural and non-trivial way. Two perspectives seem to be most promising: Considering immersed ribbon surfaces one might hope to derive lower bounds for the ribbon genus. Turning to embedded surfaces one might try to reconcile the classical approach of Seifert surfaces with finite type invariants. Here Vassiliev-Goussarov invariants are known to be too restrictive, see Murakami-Ohtsuki [38].

In analogy with the tangle category modelling knots and links, one can construct a category modelling ribbon surfaces. Once we have a presentation of this category by generators and relations, we can look for representations and extract invariants. Quite plausibly some of the extensively studied quantum representations extend to this setting, and the introduction of surfaces might reveal more topological features.

\section{References}

[1] J W Alexander, Topological invariants of knots and links, Trans. Amer. Math. Soc. 30 (1928) 275-306 MR1501429

[2] D Bar-Natan, On the Vassiliev knot invariants, Topology 34 (1995) 423-472 MR1318886

[3] D Bar-Natan, Khovanov's homology for tangles and cobordisms, Geom. Topol. 9 (2005) 1443-1499 MR2174270

[4] J S Birman, X-S Lin, Knot polynomials and Vassiliev's invariants, Invent. Math. 111 (1993) 225-270 MR1198809

[5] G Burde, H Zieschang, Knots, de Gruyter Studies in Mathematics 5, Walter de Gruyter \& Co., Berlin (1985) MR808776

[6] A J Casson, C M Gordon, Cobordism of classical knots, from: "À la recherche de la topologie perdue”, Progr. Math. 62, Birkhäuser, Boston (1986) 181-199 MR900252 With an appendix by P M Gilmer

[7] T D Cochran, Concordance invariance of coefficients of Conway's link polynomial, Invent. Math. 82 (1985) 527-541 MR811549

[8] J H Conway, An enumeration of knots and links, and some of their algebraic properties, from: "Computational Problems in Abstract Algebra (Proc. Conf., Oxford, 1967)", Pergamon, Oxford (1970) 329-358 MR0258014 
[9] M Eisermann, A geometric characterization of Vassiliev invariants, Trans. Amer. Math. Soc. 355 (2003) 4825-4846 MR1997586

[10] M Eisermann, Finite type invariants of surfaces in 3-space, in preparation (2008)

[11] M Eisermann, C Lamm, A refined Jones polynomial for symmetric unions (2008) arXiv:0802.2283

[12] D Erle, Quadratische Formen als Invarianten von Einbettungen der Kodimension 2, Topology 8 (1969) 99-114 MR0238300

[13] V Florens, On the Fox-Milnor theorem for the Alexander polynomial of links, Int. Math. Res. Not. (2004) 55-67 MR2040323

[14] R H Fox, Some problems in knot theory, from: "Topology of 3-manifolds and related topics (Proc. The Univ. of Georgia Institute, 1961)", Prentice-Hall, Englewood Cliffs, N.J. (1962) 168-176 MR0140100

[15] R H Fox, J W Milnor, Singularities of 2-spheres in 4-space and cobordism of knots, Osaka J. Math. 3 (1966) 257-267 MR0211392

[16] P Freyd, D Yetter, J Hoste, W B R Lickorish, K Millett, A Ocneanu, A new polynomial invariant of knots and links, Bull. Amer. Math. Soc. (N.S.) 12 (1985) 239-246 MR776477

[17] S Garoufalidis, Signatures of links and finite type invariants of cyclic branched covers, from: “Tel Aviv Topology Conference: Rothenberg Festschrift (1998)”, Contemp. Math. 231, Amer. Math. Soc. (1999) 87-97 MR1705577

[18] C M Gordon, Ribbon concordance of knots in the 3-sphere, Math. Ann. 257 (1981) 157-170 MR634459

[19] M N Gusarov, A new form of the Conway-Jones polynomial of oriented links, Zap. Nauchn. Sem. Leningrad. Otdel. Mat. Inst. Steklov. (LOMI) 193 (1991) 4-9, 161 MR1157140 English translation in [47]

[20] M Jacobsson, An invariant of link cobordisms from Khovanov homology, Algebr. Geom. Topol. 4 (2004) 1211-1251 MR2113903

[21] V F R Jones, A polynomial invariant for knots via von Neumann algebras, Bull. Amer. Math. Soc. (N.S.) 12 (1985) 103-111 MR766964

[22] L H Kauffman, On knots, Annals of Mathematics Studies 115, Princeton University Press, Princeton, NJ (1987) MR907872

[23] L H Kauffman, State models and the Jones polynomial, Topology 26 (1987) 395-407 MR899057

[24] L H Kauffman, An invariant of regular isotopy, Trans. Amer. Math. Soc. 318 (1990) 417-471 MR958895

[25] A Kawauchi, On the Alexander polynomials of cobordant links, Osaka J. Math. 15 (1978) 151-159 MR0488022 
[26] M Khovanov, A categorification of the Jones polynomial, Duke Math. J. 101 (2000) 359-426 MR1740682

[27] M Khovanov, An invariant of tangle cobordisms, Trans. Amer. Math. Soc. 358 (2006) 315-327 MR2171235

[28] R Kirby, editor, Problems in low-dimensional topology, from: “Geometric topology (Athens, GA, 1993)”, AMS/IP Stud. Adv. Math. 2 (1997) MR1470751

[29] S Lang, Algebra, third edition, Graduate Texts in Mathematics 211, Springer, New York (2002) MR1878556

[30] C Lescop, Global surgery formula for the Casson-Walker invariant, Annals of Mathematics Studies 140, Princeton University Press, Princeton, NJ (1996) MR1372947

[31] J Levine, Knot cobordism groups in codimension two, Comment. Math. Helv. 44 (1969) 229-244 MR0246314

[32] W B R Lickorish, An introduction to knot theory, Graduate Texts in Mathematics 175, Springer, New York (1997) MR1472978

[33] C Livingston, A survey of classical knot concordance, from: "Handbook of knot theory”, Elsevier B. V., Amsterdam (2005) 319-347 MR2179265

[34] J W Milnor, Infinite cyclic coverings, from: "Conference on the Topology of Manifolds (Michigan State Univ., E. Lansing, Mich., 1967)”, Prindle, Weber \& Schmidt, Boston (1968) 115-133 MR0242163

[35] Y Mizuma, Ribbon knots of 1-fusion, the Jones polynomial, and the Casson-Walker invariant, Rev. Mat. Complut. 18 (2005) 387-425 MR2166517 With an appendix by Tsuyoshi Sakai

[36] Y Mizuma, An estimate of the ribbon number by the Jones polynomial, Osaka J. Math. 43 (2006) 365-369 MR2262340

[37] D Mullins, The generalized Casson invariant for 2-fold branched covers of $S^{3}$ and the Jones polynomial, Topology 32 (1993) 419-438 MR1217078

[38] H Murakami, T Ohtsuki, Finite type invariants of knots via their Seifert matrices, Asian J. Math. 5 (2001) 379-386 MR1868640

[39] H Murakami, T Ohtsuki, S Yamada, Homfly polynomial via an invariant of colored plane graphs, Enseign. Math. (2) 44 (1998) 325-360 MR1659228

[40] K Murasugi, On a certain numerical invariant of link types, Trans. Amer. Math. Soc. 117 (1965) 387-422 MR0171275

[41] G Polya, Induction and analogy in mathematics. Mathematics and plausible reasoning, vol. I, Princeton University Press, Princeton, N. J. (1954)

[42] J H Przytycki, P Traczyk, Invariants of links of Conway type, Kobe J. Math. 4 (1988) 115-139 MR945888 
[43] J A Rasmussen, Khovanov homology and the slice genus, Invent. Math. (to appear) arXiv:math/0402131

[44] D Rolfsen, Knots and links, Mathematics Lecture Series 7, Publish or Perish, Houston, TX (1990) MR1277811 Corrected reprint of the 1976 original

[45] A G Tristram, Some cobordism invariants for links, Proc. Cambridge Philos. Soc. 66 (1969) 251-264 MR0248854

[46] V A Vassiliev, Cohomology of knot spaces, from: "Theory of singularities and its applications”, Adv. Soviet Math. 1, Amer. Math. Soc. (1990) 23-69 MR1089670

[47] O Viro, editor, Topology of manifolds and varieties, Advances in Soviet Mathematics 18, Amer. Math. Soc. (1994)

Institut Fourier, Université Grenoble I, France

Michael.Eisermann@ujf-grenoble.fr

http://www-fourier.ujf-grenoble.fr/ eiserm

Proposed: Joan Birman

Seconded: Peter Teichner, Ron Stern

Received: 19 February 2008

Revised: 28 July 2008 